\title{
Floristic study of Saint Katherine protectorate, Sinai: with one new record to flora of Egypt.
}

\author{
Sami Rabei ${ }^{1 *}$, Abdel-Hamid Khedr ${ }^{1}$ and Ibrahim Elgamal ${ }^{2}$
}

\author{
${ }^{1}$ Botany and Microbiology Department, Faculty of Science, Damietta University, New Damietta, Egypt \\ ${ }^{2}$ Saint Katherine Protectorate, Egyptian Environmental Affairs Agency (EEAA), Egypt \\ *Corresponding author: samirabei@du.edu.eg
}

\section{Abstract}

Saint Katherine protectorate is one of the most floristically diverse spots in the Middle East. A field surveys, as well as critical revision of herbarium specimens, were carried out during 2013-2020 revealed that 430 taxa belonging to 283 genera and 62 families. The life-forms are classified into seven life forms with dominance of Therophytes (39\%) followed by Chamaephyte (24.7\%). Chorological study indicated that $52 \%$ of recorded taxa were monoregional native to Saharo-Arabian (29\%), Irano-Turanian (10.39\%), Mediterranean (7.4\%), Sudanin (4\%) while tropical and sub- tropical represented by (0.2\%) only. 223 taxa were mono-regional, 127 taxa were bi-regional, while 16 taxa were endemic and Stenoendemic. Investigated taxa distributed in four microhabitats; terraces, slopes, gorges, and wadi bed the last two were the most diversity. The present study investigated that presence of Papaver somniferum is a new record to flora of Egypt, eight new records to Sinai and thirty four new records to the study area.

Keywords: Chorology, life forms, new record, Saint Katherine Protectorate

\section{Introduction}

Floristic composition is a reflection of plant diversity of a geographic region (Rafay et al. 2013); a floristic inventory is the main source of botanical information and provides an appropriate starting point for supplementary comprehensive studies of a specific geographic area (Keith 1988) . Plant life form as the growth form that represents an adaptation to specific environmental conditions (Galán de Mera et al. 1999) can reflect the climatic adaptability and then the whole vegetation in a particular ecosystem would be an expression of the prevailing climate (Batalha et al., 2002) .

Sinai Peninsula is the meeting place of the old world (Grainger 2003), it occupies $6.1 \%$ of the surface of Egypt; it is rich both in the number of species and the high percentage of endemics as it hosts 1262 taxa (Boulos 2009). According to (Zahran and Willis 2009) Sinai Peninsula stands in four phytogeographical regions: Mediterranean, Irano-Turanian, Sudano-Deccanian and Saharo - Arabian. The majority of Sinai belongs to the Sahara-Arabian phytogeographical region. Its vegetation is characterized by sparseness vegetation; while the high mountains of southern Sinai support mainly Irano-Turanian vegetation. (Danin Avinoan 1986) .

Saint Katherine Protectorate (SKP) (sometimes spelled as Saint Catherine protectorate) spreads over virtually the entire mountain massif of South Sinai, with an area of about $4350 \mathrm{~km}^{2}$. and lies between $33^{\circ} 55^{\prime}$ to $34^{\circ} 30^{\prime} \mathrm{E}$ and $28^{\circ} 30^{\prime}$ to $28^{\circ} 35^{\prime} \mathrm{N}$ 
with an elevation up to $2641 \mathrm{~m}$ above sea level (asl); it lies in a SaharanMediterranean climate with extremely dry, hot summers and cold winters; average rainfall is $60 \mathrm{~mm}$ / year, It is the coolest area in Egypt. Mean maximum temperature in August is $36^{\circ}$ while winters are relatively cool with mean minimum temperature of $7.8^{\circ}$ in February (Grainger 2003). SKP is one of the most floristically diverse spots in the Middle East (Boulos 2009) with a significant proportion of endemic species as it hosts 17 endemic taxa (Ghaly 2015) out of them 6 are endangered and /or critically endangered; the majority of SKP endemic taxa classified as steno-endemic (Ghaly 2015; Hosni et al. 2013) The diversity of both landforms and geologic structures of SKP leads to the differentiation of a six microhabitats namely; farsh (Basine), caves, terraces, slopes, wadis and gorges the last two microhabitats have dykes that trap water resulting in significant plant cover (Khedr 2007).

According to (Fayed et al. 2004) and (Shaltout et al. 2004) the total number of taxa in Saint Katherine protectorate is 472, belonging to 272 genera and 64 families, (Fayed et al. 2010) carried out Partial Survey in SKP; and listed 134 species belonging to 117 genera and representing 38 families. Since this date there is no partial or whole survey throughout SKP even if there are many climatic changes, human impacts and threats (Omar et al. 2015).

The authors aim to (1) carrying out field survey in an area where few floristic studies had been done before, (2) documented chorological affinities and life spans (3) confirmation the presence of Papaver somniferum L. as a new record in flora of Egypt, (4) reporting 8 new taxa to Sinai, and 34 new taxa to SKP.

\section{Material and methods}

An inventory of all available specimens was compiled based on field surveys that carried out in Saint Katherine Protectorate, South Sinai, Egypt during 2013-2020 as well as critical revision of herbarium specimens hosted in SKP herbarium, collected taxa were identified according to (Feinbrun-Dothan 1986, 1978; Täckholm 1974; Zohary, 1973, 1966; Boulos, 2005, 2002, 2000, 1999). Таха names were reconciled against Kew's Plants of the World Online (POWO 2021) which was also used as reference for families and genera classification. For the few species that were not found in POWO the authors followed (GBIF 2021).

Voucher specimens were kept at Saint Katherine Protectorate Herbarium (SKPH) without identification numbers. Life forms were followed (Raunkiaer, 1934), when several life forms were given for a taxon, the most representative one was chosen; variation in the life form in the field was not considered, phytogeographical affinity, the general approach and terminology of (Zohary 1973) for the Saharo-Arabian and Sudanian regions, each species Phytogeographical affinity was obtained from (Abd El-Ghani 1985, 1981); when these resources for a single taxon gave more than one phytogeographical element, the most appropriate was used, vegetation type and floristic division according to (Boulos 2005, 2002, 2000, 1999). The specimens were first checked in (Boulos 2005, 2002, 2000, 1999; Fayed et al. 2004; Moustafa et al. 1998; Shaltout et al. 2004; Täckholm 1974) ,for their presence in Egypt, Sinai and SKP, rarity and endemism based on IUCN red list 2018 https://www.iucnredlist.org, (Omar et al. 2015; Walter and Harriet 1997). 


\section{Floristic study of Saint Katherine protectorate, Sinai}

Density, frequency, and different diversity indices calculation

Shannon index was calculated using the formula $H^{\prime}=-\Sigma$ PilnPi (Pielou 1975).The relative concentration of dominance within each plot was assessed by the Simpson diversity index $D=1-\Sigma n(n i-1) / N(N-1)$. Species richness was calculated as the average number of species/ sampling plot. Density of species (i) is calculated as the total number of individuals of species (i) in all sampled plots/ area of sampled plots. Frequency is the number of times a plant species occurs in a given number of quadrats. One-way ANOVA was performed to calculate the significance of differences in diversity indices, both average density and frequency between different quadrates that varied in different microhabitats using SPSS software version 22.

\section{Results and discussion}

\section{Floristic composition}

Despite its small area, SKP region has high floristic richness as it hosts approximately 23\% of Egyptian flora (Fayed et al. 2004; Shaltout et al. 2004) and 28\% of Egyptian endemic taxa (Ghaly 2015), a total of 430 taxa including 27 infraspecific from the study area were identified; belonging to 283 genera and 62 families(see Appendix 1) of these Papaver somniferum is a new record to flora of Egypt, eight taxa are new records to Sinai and thirty four are new records to Saint Katherine Protectorate in addition to sixteen endemic taxa (table 1) .

Table (1) Endemic and new records taxa, SKP= Saint Katherine Protectorate

\begin{tabular}{|c|c|c|}
\hline Accepted plant name & Family & Status \\
\hline Anarrhinum forskaohlii subsp. pubescens D.A. Sutton & Plantaginaceae & Endemic \\
\hline Ballota kaiseri Täckh & Lamiaceae & Endemic \\
\hline Bufonia multiceps Decne. & Caryophyllaceae & Endemic \\
\hline Euphorbia obovata Decne. & Euphorbaiceae & Endemic \\
\hline Hyoscyamus boveanus (Dunal) Asch. \& Schweinf. & Solanaceae & Endemic \\
\hline Micromeria serbaliana Danin \& Hedge & Lamiaceae & Endemic \\
\hline Origanum syriacum subsp. Sinaicum (Boiss.) Greater \& Burdet & Lamiaceae & Endemic \\
\hline Phlomis aurea Decne. & Lamiaceae & Endemic \\
\hline Polygala Sinaica Botsch. & Polygalaceae & Endemic \\
\hline Primula involucrata (Raf.) Link \& Otto ex Sweet & Primulaceae & Endemic \\
\hline Pterocephalus arabicus Boiss. & Caprifoliaceae & Endemic \\
\hline Rosa arabica (Crép. ex Boiss.) Déségl. & Rosaceae & Endemic \\
\hline Silene leucophylla Boiss. & Caryophyllaceae & Endemic \\
\hline Silene oreosinaica Chowdhuri & Caryophyllaceae & Endemic \\
\hline Silene schimperiana Boiss. & Caryophyllaceae & Endemic \\
\hline Veronica kaiseri Täckh. & Plantaginaceae & Endemic \\
\hline Achillea tenuifolia Lam. & Asteracea & New record to SKP \\
\hline Arabis recta Vill. & Brassicaceae & New record to SKP \\
\hline Argyrolobium arabicum (Decne.) Jaub. \& Spach. & Fabaceae & New record to SKP \\
\hline Astragalus amalecitanus Boiss. & Fabaceae & New record to SKP \\
\hline Astragalus echinus DC. & Fabaceae & New record to SKP \\
\hline Astragalus palaestinus Eig. & Fabaceae & New record to SKP \\
\hline Capsella bursa-pastoris (L.) Medik & Brassicaceae & New record to Sinai \& SKP \\
\hline Caudanthera Sinaica (Decne.) Plowes & Apocynaceae & New record to SKP \\
\hline Centaurium pulchellum (Sw.) Druce. & Gentianaceae & New record to SKP \\
\hline Cistanche salsa (C.A. Mey.) Beck & Orobanchaceae & New record to SKP \\
\hline Cleome chrysantha Decne. & Cleomiaceae & New record to SKP \\
\hline Clinopodium barbatum (P.H. Davis) Melnikov & Lamiaceae & New record to Sinai \& SKP \\
\hline Colchicum ritchii $\mathrm{R} . \mathrm{Br}$. & Colchicaceae & New record to SKP \\
\hline
\end{tabular}




\section{Rabei et al.}

\begin{tabular}{lll}
\hline Accepted plant name & Family & \multicolumn{1}{c}{ Status } \\
\hline Datura ferox L. & Solanaceae & New record to Sinai \& SKP \\
Dianthus libanotis Labill. & Caryophyllaceae & New record to SKP \\
Euphorbia granulata Forssk & Euphorbaiceae & New record to SKP \\
Euphorbia hirta L. & Euphorbaiceae & New record to SKP \\
Glebionis coronaria (L.) Tzvelev & Asteracea & New record to SKP \\
Lamium amplexicaule L. & Lamiaceae & New record to SKP \\
Limonium lobatum (L. fil.) Chaz. & Plumbaginaceae & New record to SKP \\
Limonium sinuatum (L.) Mill. & Plumbaginaceae & New record to Sinai \& SKP \\
Lotus hebranicus Hochst. ex Brand & Fabaceae & New record to SKP \\
Nidorella aegyptiaca (L.) J.C. Manning \& Goldblatt & Asteracea & New record to Sinai \& SKP \\
Orobanche palaestina Reuter & Orobanchaceae & New record to Sinai \& SKP \\
Pallenis spinosa (L.) Cass. & Asteracea & New record to SKP \\
Papaver somniferum L. & Papaveraceae & New record to Egypt, Sinai \& SKP \\
Piptatherum holciforme (M. Bieb.) Roem. \& Schult. & Poaceae & New record to SKP \\
Spergularia marina (L.) Griseb. & Caryophyllaceae & New record to SKP \\
Stellaria media (L.) Vill. & Caryophyllaceae & New record to SKP \\
Tribulus pentandrus Forssk. & Zygophyllaceae & New record to SKP \\
Tricholaena teneriffae (L. f.) Link. & Poaceae & New record to SKP \\
Vicia articulata Hornem & Fabaceae & New record to Sinai \& SKP \\
Withania somnifera (L.) Dunal & Solanaceae & New record to SKP \\
Ziziphora tenuior L. & Lamiaceae & New record to SKP \\
\hline
\end{tabular}

The surveyed flora represented by Angiospermae (Dicotyledoneae 363 taxa, and Monocotyledoneae 61 taxa) while Gymnospermae and Pteridophyta represented by three species for each. More than $60 \%$ of the recorded taxa belong to only 10 species rich families. The largest families were Asteraceae, Poaceae and Fabaceae while nine families were represented by two species, and the remaining twenty-three families were represented by one species only (Fig 1).

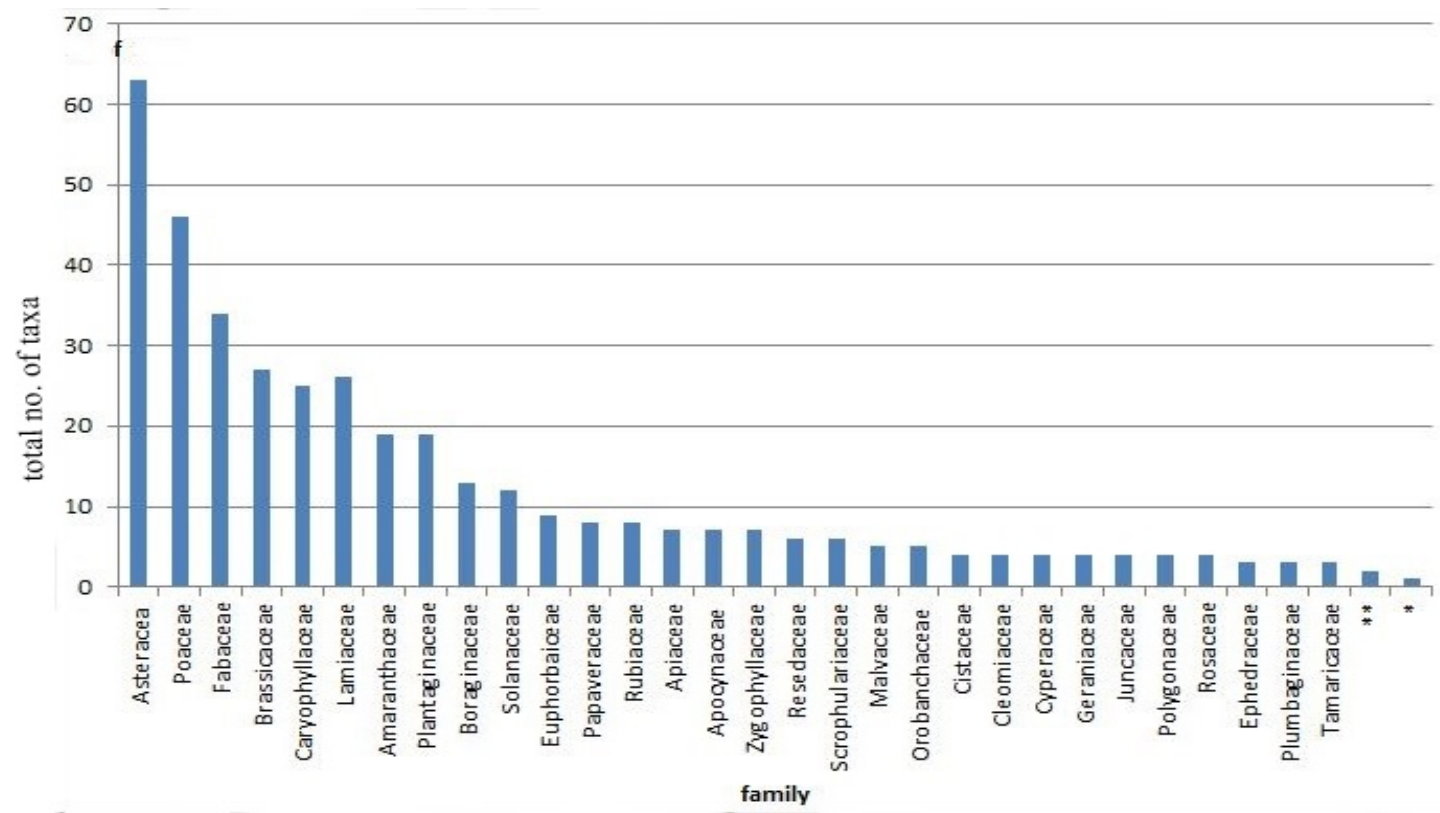

Fig (1) Representative number of taxa of the recorded families; * = 23 families represented by one taxon, and $* *=9$ families represented by two taxa. 


\section{Floristic study of Saint Katherine protectorate, Sinai}

Investigated taxa classified into herbaceous plants (301 taxa); shrubs (92 taxa); sub Shrubs (16 taxa), trees (12 taxa), Climber (5 taxa), reeds and Herb/ Subshrub represented by 2 taxa for each (Fig 2), the dominance of herbaceous taxa over the other growth habits can be recognized to their short life cycle that enables them to resist the instability of the harsh environmental condition (Gomaa 2012) .

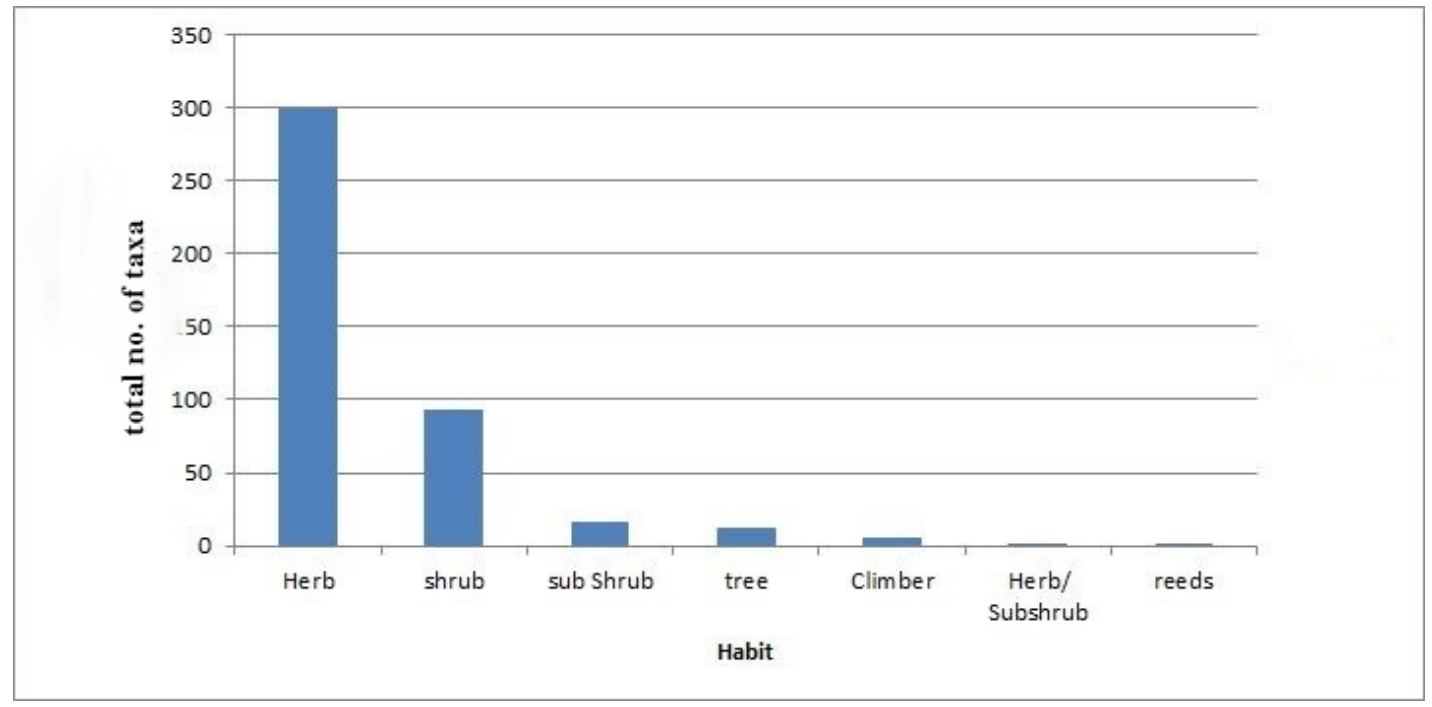

Fig (2) Number of taxa in relation to their habit

\section{Life spans and Life forms}

Investigated taxa classified into six groups dominant by Perennial (234 taxa), annual (173 taxa), annual / short perennial (13 taxa), annual/ biennial and biennial represented by four taxa for each and biennial/ perennial represented only by one taxon (Fig 3).

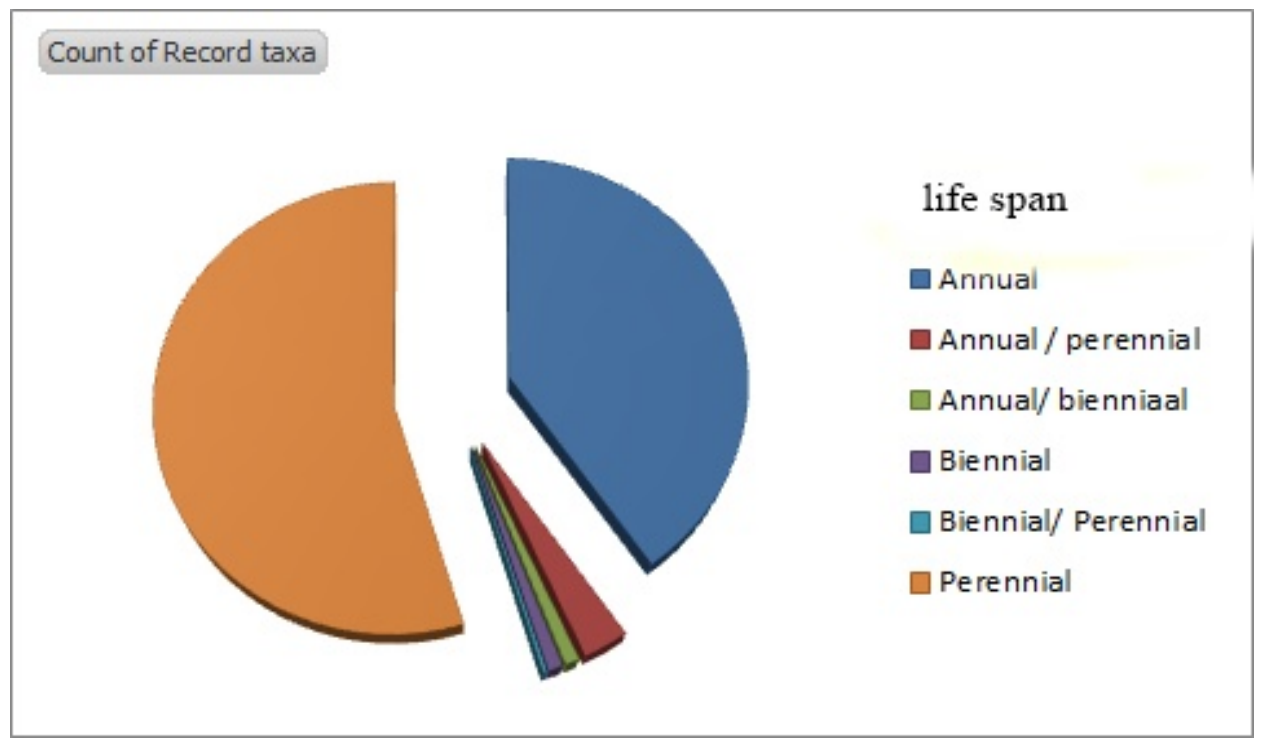

Fig (3) life span of the recorded taxa 


\section{Rabei et al.}

Life forms spectrum is characteristic of an arid ecosystem with the dominance of therophytes (171) followed by chamaephyte (106 taxa), hemicryptophyte (102 taxa), phanerophyte (34 taxa), geophyte (11 taxa), parasite (4 taxa) and helophyte represented only by two species (Fig 4), with agreement with previous studies e.g (Elgamal 2020; Moustafa and Zaghloul 1996) Life-form spectrum in our result is typical of an arid desert region with the dominance of therophyes and this support the concept of (Cain 1950; Deschenes 1969) that arid conditions and overgrazing are so prevalent on grasslands tend to increase therophytes percentage through the introduction and spread of weedy grasses and forbs of this life form.

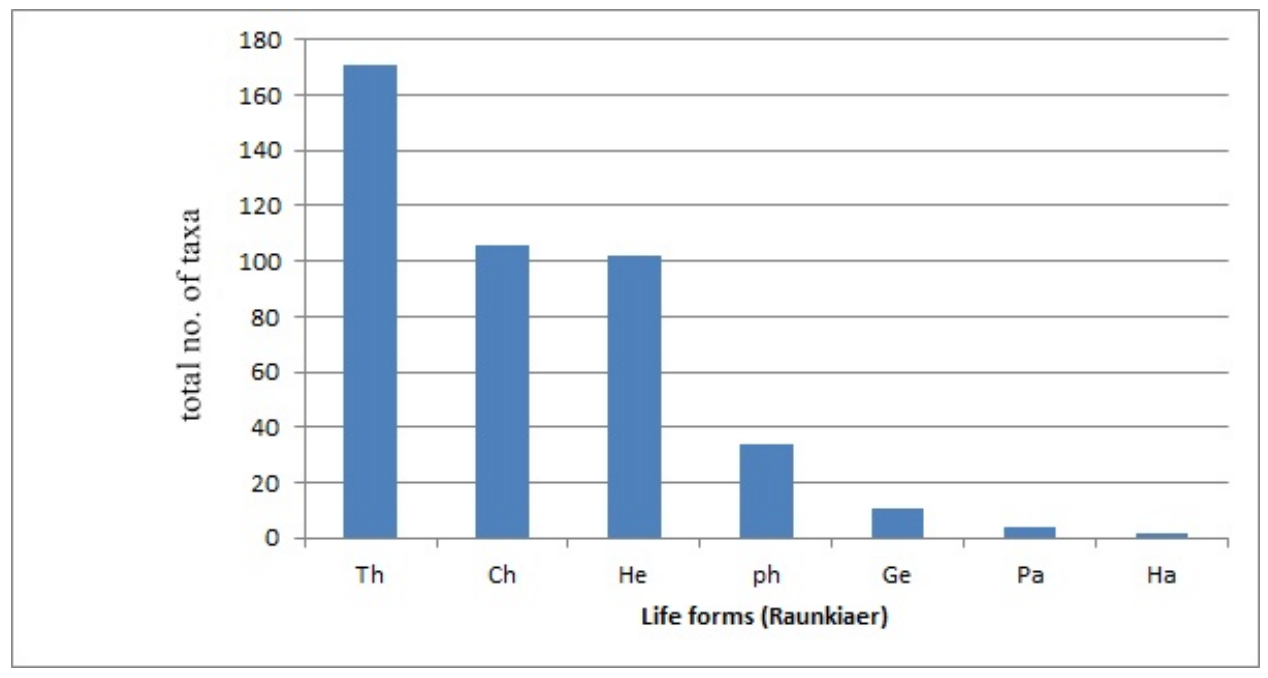

Fig (4) Life forms of the recorded taxa, $\mathrm{He}=$ Hemicryptophyte, $\mathrm{Ch}=$ chamaephyte, $\mathrm{Th}=$ Therophytes, $\mathrm{Ph}=$ Phaneroohyte, $\mathrm{Ge}=$ Geophyte, $\mathrm{Pa}=$ Parasite and $\mathrm{Ha}=$ Helophyte.

\section{Phytogeographical affinities}

Surveyed taxa related to 6 phytogeographical regions thus the Mediterranean region, Saharo - Arabian, Irano-Turanian, Sudano - Deccanian, Tropical and sub-tropic region. The analysis of surveyed flora chorology revealed that $52 \%$ taxa were monoregional native to Saharo - Arabian (29\%), Irano- Turanian
(10.39\%), Mediterranean (7.39\%), Sudanin (4\%) while tropical and sub- tropical represented by $(0.2 \%)$ only. 127 taxa were bi-regional, 63 taxa were recognized in more than two phytogeographical regions and/ or pluri- regional while 16 were endemic and Steno-endemic (Fig 5). 


\section{Floristic study of Saint Katherine protectorate, Sinai}

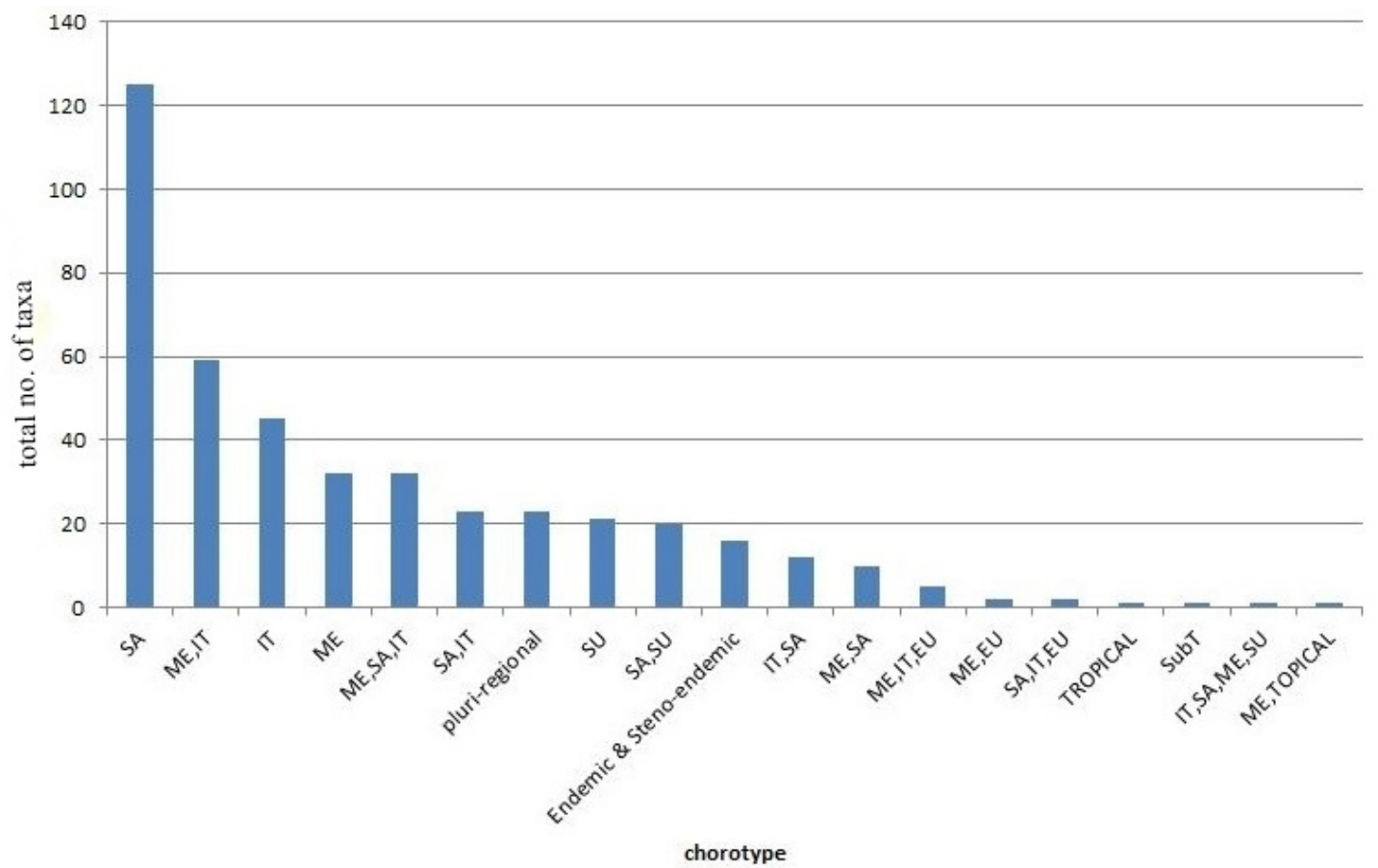

Fig (5) Chorological analysis of the recorded taxa ME = Mediterranean, IT = Irano- turanian, SA = Saharo - Arabian, $\mathrm{SU}=$ Sudanin, and Sub $\mathrm{T}=$ sub trobic

Concerning the relation between geoelements and life forms (Table 2), chamaephytes followed by therophyte; hemicryptophytes and phanerophytes make an important contribution to the SaharoArabian and Irano-Turanian phtogeographical regions, while halophytes and parasites were completely absent. Phanerophyte layer is composed mainly of both Saharo- Arabian and SudanoDeccanian geoelement. The Mediterranean geoelement was humbly represented in the tree and shrub layer. That may be recognized to the fact that the SaharoArabian and Sudano geoelements are good indicators for harsh ecosystems (Abd ElGhani and Abdel-Khalik 2006).

Table (2) Distribution of the geoelements among life forms He = Hemicryptophyte, $\mathrm{Ch}=$ chamaephyte, Th = Therophytes, $\mathrm{Ph}=$ Phaneroohyte, $\mathrm{Ge}=$ Geophyte, $\mathrm{Pa}=$ Parasite and He $=$ Helophyte $\mathrm{ME}=$ Mediterranean, IT = Irano - Turanian, SA = Saharo - Arabian, SU = Sudanin, and Sub T = sub trobic. Other taxa are distributed in more than two regions

\begin{tabular}{ccccccccccc}
\hline Life form & SA & IT & ME & SU & Sub T & T & Endemic & Bi- regional & Others & total \\
\hline $\mathrm{Th}$ & 36 & 14 & 14 & 5 & 1 & 1 & 0 & 58 & 42 & 171 \\
$\mathrm{Ch}$ & 49 & 15 & 6 & 5 & 0 & 0 & 4 & 22 & 5 & 106 \\
$\mathrm{He}$ & 27 & 12 & 10 & 3 & 0 & 0 & 11 & 30 & 9 & 102 \\
$\mathrm{Ph}$ & 9 & 3 & 1 & 8 & 0 & 0 & 1 & 11 & 1 & 34 \\
$\mathrm{Ge}$ & 3 & 0 & 1 & 0 & 0 & 0 & 0 & 3 & 4 & 11 \\
$\mathrm{~Pa}$ & 0 & 1 & 0 & 0 & 0 & 0 & 0 & 1 & 2 & 4 \\
$\mathrm{Ha}$ & 0 & 0 & 0 & 0 & 0 & 0 & 0 & 0 & 2 & 2 \\
\hline
\end{tabular}




\section{Rabei et al.}

\section{Microhabitat diversity and abundance}

Based on Khedr (2007), six microhabitats can be recognized throughout SKP these are wadi bed, terraces, slopes, gorges, farsh and caves. Investigated taxa are distributed mainly in four out of these six microhabitats viz. wadi, terraces, gorges and slopes with limited availability to farsh and caves microhabitats. The distribution of plant species was higher in gorges; wadi bed as well as north aspect compared to the other microhabitats and aspect. In general, Shannon index, richness, frequency and density were significantly higher in gorges and wadi bed compared to the other microhabitats. Similarly, the concentration of dominance, as indicated by the Simpson index, with non-significant effect $(P>0.05)$. (Table 3).

Table (3). Results of one-way ANOVA of species density, frequency, and different diversity indices at different microhabitat

\begin{tabular}{lccccc}
\hline & Goreoge & Slope & Terraces & Wadi & F value \\
\hline Shannon index & 2.06 & 1.5 & 1.7 & 1.8 & 3.8 \\
Simpson diversity index & 0.873 & 0.85 & 0.87 & 0.89 & 648 \\
Richness & 10.8 & 6.2 & 7.7 & 10.2 & 7.5 \\
Frequency & 18.22 & 11.97 & 10.99 & 15.02 & 4.0 \\
density & 15.4 & 9.4 & 14.08 & 34.3 & 7.2 \\
\hline
\end{tabular}

Goreoge ,wadi bed and north aspect sites were the most rich microhabitat and aspect while hilltops and south-facing slopes, which will be exposed to more intense insolation, will be the harshest habitats for plant growth, it seems that soil moisture and evaporation rate are among environmental factors determinants influencing species composition and richness in the arid habitat; as Goreoge and wadis have the greater merit of being a drainage system collecting water from great catchment area (El Hadidi 2000; Khedr, 2007) as well as the lowest evaporation rate (Kidron and Zohar 2010), Altitude and aspect on mountains influence the amount of water available to plants (Lomolino 2001); soils at higher altitudes dry out quickly after rain events; this can lead to very dry soils through the rest of the year. (Kidron and Zohar 2010) studied the evaporation rates in different parts of the slope in a drainage basin in the Negev Desert Highlands; they ranked Sites in the following order: northfacing aspect $<$ wadi beds $\leq$ west-facing aspect $\leq$ east-facing aspect $\leq$ south-facing
Aspect < hilltops. As water is the most limiting factor for plant growth and survival in arid regions, the wadi beds and goreoges provides the most favorable conditions for plant growth.

\section{Species that should receive more priority for conservation}

According to the species endemism, are these species listed on the Red List of the International Union of Conservation (IUCN), density and fraquency of these species, threats to plant species, the present study concluded that twenty five taxa classified as rare (R), with two taxa recently added to Egyptian flora as one population (Table 4); out of them sixteen species are endemic and / or steno-endemic to SKP; according to IUCN red-list six species are endangered or critically endangered. The rarity and endemism of these species indicate that they may be threatened by biotic and abiotic factors and should receive a high priority for conservation. 


\section{Floristic study of Saint Katherine protectorate, Sinai}

Table (4) Species that should receive more priority for conservation, R= rare, VU= vulnerable, EN= endangered, $\mathrm{CR}=$ critically endangered

\begin{tabular}{|c|c|c|c|c|}
\hline Record taxa & family & Global distribution & $\begin{array}{l}\text { Conservation } \\
\text { status }\end{array}$ & $\begin{array}{l}\text { IUCN red list } \\
\text { category(2018) }\end{array}$ \\
\hline $\begin{array}{l}\text { Anarrhinum forskaohlii subsp. pubescens D.A. } \\
\text { Sutton* }\end{array}$ & Plantaginaceae & Endemic & $\mathrm{R}$ & EN \\
\hline Ballota kaiseri Täckh & Lamiaceae & Endemic & $\mathrm{R}$ & Not evaluated \\
\hline Bufonia multiceps Decne & Caryophyllaceae & Endemic & $\mathrm{R}$ & EN \\
\hline Clinopodium barbatum (P.H.Davis) Melnikov & Lamiaceae & $\begin{array}{l}\text { Lebanon-Syria; Palestine; } \\
\text { Turkey, recently added to flora } \\
\text { of Egypt by Rabei et al (2019) } \\
\text { as one population }\end{array}$ & VU & Not evaluated \\
\hline Datura ferox L. & Solanaceae & $\begin{array}{l}\text { World wide, recently added to } \\
\text { flora of Egypt by Rabei et al } \\
\text { (2019)as one population }\end{array}$ & Not evaluated & Not evaluated \\
\hline Dianthus Sinaicus Boiss. & Caryophyllaceae & $\begin{array}{l}\text { Egypt and Palestine } \\
\text { (near endemic) }\end{array}$ & VU & Not evaluated \\
\hline Euphorbia obovata Decne. & Euphorbaiceae & Endemic & $\mathrm{R}$ & EN \\
\hline $\begin{array}{l}\text { Hyoscyamus boveanus (Dunal) Asch. \& } \\
\text { Schweinf. }\end{array}$ & Solanaceae & Endemic & $\mathrm{R}$ & Not evaluated \\
\hline Hypericum Sinaicum Hochst. ex Boiss & Hypericaceae & $\begin{array}{l}\text { Egypt and Saudi Arabia } \\
\text { (near endemic) }\end{array}$ & $\mathrm{R}$ & Not evaluated \\
\hline Micromeria serbaliana Danin \& Hedge & Lamiaceae & Endemic & $\mathrm{R}$ & Not evaluated \\
\hline Micromeria Sinaica Benth. & Lamiaceae & $\begin{array}{l}\text { Egypt and Palestine } \\
\text { (near endemic) }\end{array}$ & $\mathrm{R}$ & Not evaluated \\
\hline Nepeta septemcrenata Benth. & Lamiaceae & $\begin{array}{l}\text { Egypt and Saudi Arabia } \\
\text { (near endemic) }\end{array}$ & $\mathrm{R}$ & Not evaluated \\
\hline $\begin{array}{l}\text { Origanum syriacum subsp. sinaicum (Boiss.) } \\
\text { Greater \& Burdet. }\end{array}$ & Lamiaceae & Endemic & $\mathrm{R}$ & Not evaluated \\
\hline Phlomis aurea Decne. & Lamiaceae & Endemic & $\mathrm{R}$ & EN \\
\hline Plantago sinaica (Barneoud) Decne. & Plantagonaceae & $\begin{array}{l}\text { Egypt and Palestine } \\
\text { (near endemic) }\end{array}$ & $\mathrm{R}$ & Not evaluated \\
\hline Polygala sinaica Botsch. & Polygalaceae & Endemic & $\mathrm{R}$ & Not evaluated \\
\hline Primula involucrata (Raf.) Link \& Otto ex Sweet & Primulaceae & Endemic & $\mathrm{R}$ & $\mathrm{CR}$ \\
\hline Pterocephalus arabicus Boiss & Caprifoliaceae & Endemic & VR & Not evaluated \\
\hline Rosa arabica (Crép. ex Boiss.) Déségl. & Rosaceae & Endemic & $\mathrm{R}$ & $\mathrm{CR}$ \\
\hline Silene leucophylla Boiss. & Caryophyllaceae & endemic & $\mathrm{R}$ & Not evaluated \\
\hline Silene odontopetala subsp congesta Boiss. & Caryophyllaceae & Egypt and Iran & $\mathrm{R}$ & Not evaluated \\
\hline Silene oreoSinaica Chowdhuri & Caryophyllaceae & Endemic & $\mathrm{R}$ & Not evaluated \\
\hline Silene schimperiana Boiss. & Caryophyllaceae & Endemic & $\mathrm{R}$ & Not evaluated \\
\hline Thymus decussatus Benth. & Lamiaceae & $\begin{array}{l}\text { Egypt ,Palestine; Saudi } \\
\text { Arabia(near endemic) }\end{array}$ & EN & Not evaluated \\
\hline Veronica kaiseri Täckh. & Scrophulariaceae & Endemic & $\mathrm{R}$ & Not evaluated \\
\hline
\end{tabular}

\section{New record to the flora of Egypt}

Papaver L. one of 50 genera related to family papaveraceae, characteristic by absence of style and presence of stigmatic tissue arranged radially on a sessile stigmatic disc which crowns the ovary (Kadereit, 1993). Papaver has long been at attention for many botanists because of it is content from alkaloids like Papaverin, Codein, Morphin (Sariyar 2002) . Papaver comprises 80 annual, biennial and perennial species in central and south-western Asia, central and southern Europe and northern Africa (Kadereit 1993; Kadereit et al. 1997) In Egypt Papaver represented by seven species namely P. argemone L., P. decaisnei Hochst. \& Steud. ex Boiss., P. dubium L., $P$. humile Fedde, $P$. hybridum L., $P$. 
macrostomum Boiss. \& Huet ex Boiss. and P.rhoeas L. (Boulos 2009)

Papaver somniferum L., Sp. Pl.: 508 (1753) TYPE: Lectotypified by Britton and A. Brown, Ill. Fl. N. U.S., ed. 2. 2: 136. 1913. Syn. Papaver setigerum auct. non DC.; P. somniferum L. var. hortense (Huss.) Corb.

Global distribution widely distributed in Europe and Asia.

Annual, 20-120 cm height. Stems erect, simple or branched, glabrous rarely bristled. Internode length 3-3.5 cm, leaves simple, alternate, oblong or ovate, toothed and lobed, rarely pinnately lobed, 4- 15 X1-
$5 \mathrm{~cm}$, leaf margin dentate or serrate. Flower solitary, terminal, Sepals glabrous, caduceus, petals 4 entire, obovate-orbicular, toothed, purple, red, violet or white, up to 5.5 -3.5 cm., Stamens long yellowish filaments fruit (capsule) dry and splits open when ripe, glabrous, ovate to oblong, sessile, and crowning the top of the ovary, stigmatic disk shorter than the capsule breadth, with deeply cut marginal lobes, seeds grey to black. Flowering and fruiting between February to June (Fig 6). Papaver somniferum can distinguished from other Egyptian papaver by its oblong or ovate stem-clasping leaves
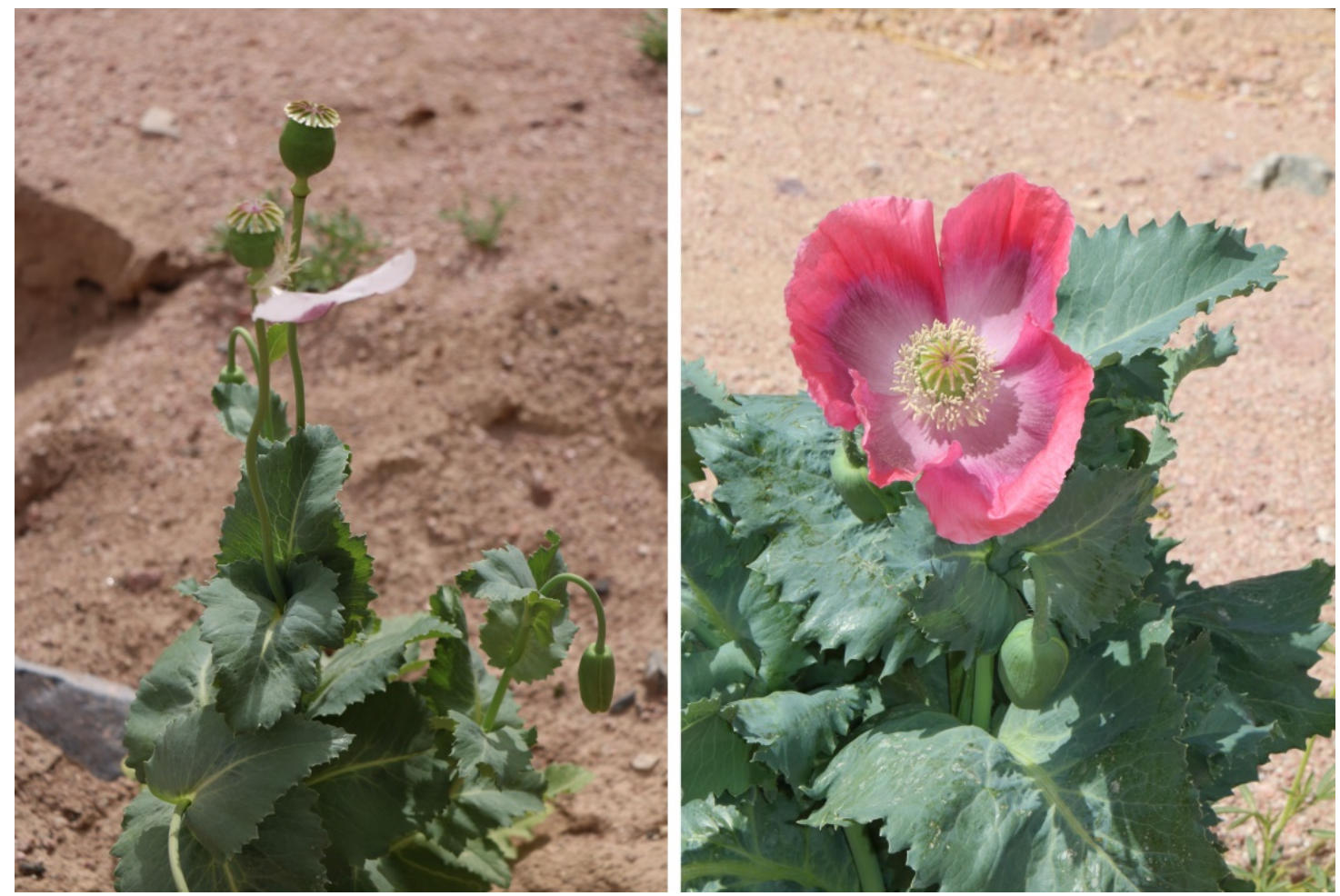

Fig (6) Papaver somniferum 


\section{References}

Abd El-Ghani, M. 1985. Comparative study on the vegetation of the Bahariya and Farafra Oases and the Faiyum region. Cairo University (Unpublished).

Abd El-Ghani, M. 1981. Preliminary studies on the vegetation of Bahariya Oasis, Egypt. Cairo unviersity (Unpublished).

Abd El-Ghani, $M$ and Abdel-Khalik, K.N., 2006. Floristic diversity and phytogeography of the Gebel Elba National Park, south-east Egypt. Turk. J. Botany 30, 121-136.

Batalha, A., Marco A and Roberto Martins, F. 2002. Life-form spectra of Brazilian cerrado sites. Flora 197, 452460.

Boulos, L. 2009. Flora of Egypt Checklist, revised annotated edition. Al Hadara Publishing Egypt., Cairo.

Boulos, L. 1999-2005. Flora of Egypt. Vols. 1-4. Al-Hadara Publishing, Cairo.

Cain, S.A. 1950. The Botan Ical Revi Ew Life-Forms and Phytoclimate XVI.

Danin Avinoan, 1986. Flora and vegetation of Sinai. Proc. R. Soc. Edinburgh. https://doi.org/10.1017/S0269727000008 99X

Deschenes, J. 1969. Life form spectra of contrasting slops of the grazed pastures of Northern New Jersey. Neturalise Can. 60, 965-978.

El Hadidi, M.N. 2000. Flora Aegyptiaca. Vol. 1. The Palm Press.

Elgamal, I. 2020. Flora of Wadi Gebal, Saint Katherine Protectorate, South Sinai: Some Additions and Taxonomic Revision. Damietta.PhD Mansoura university (unpublished).

Fayed, A., El-Garf, I., Abdel-Khalik, K. and Osman, A.E. 2004. Floristic Survey of the Mountainous Southern Sinai: Saint Katherine Protectorate (A), Conservation and Sustainable Use of Medicinal Plants in Arid and Semi-arid Eco-systems
Project, Egypt.

Fayed, A., Hassan, N., Hatab, E.-B., Faried, A. and Aboul-Ela, M. 2010. Floristic Survey of Saint Katherine's Protectorate (1-7 May 2010) Conservation and Sustainable Use of Medicinal Plants in Arid and Semi-arid Eco-systems Project, Egypt.

Feinbrun-Dothan, N. 1978, 1986. Flora Palaestina,part3- 4. The Israel Academy of Science and Humanities, Jerusalem.

Galán de Mera, A., Hagen, M.A. and Vicente Orellana, J.A. 1999. Aerophyte, a new life form in Raunkiaer's classification? J. Veg. Sci. 10, 65-68. https://doi.org/10.2307/3237161

GBIF Secretariat: GBIF Backbone Taxonomy. https://doi.org/10.15468/39omei Accessed via https://www.gbif.org/species/5284517 [20 Agust 2021]

Ghaly, O. 2015. Taxonomical and Molecular Characterization of Endemic Species at Saint Katherine ProtectorateSinai- Egypt.PhD Mansoura university (unpublished).

Gomaa, N.H. 2012. Composition and diversity of weed communities in Al-Jouf province, northern Saudi Arabia. Saudi J. Biol. Sci. 19, 369-376. https://doi.org/10.1016/j.sjbs.2012.05.002

Grainger, J. 2003. The Management and Development Plan; Saint Katherine Protectorate, a World Heritage Site, IUCN Category IV Protected Landscape.

Hosni, H., Hosny, A., Shamso, E. and Hamdy, R. 2013. Endemic and NearEndemic Taxa in the Flora of Egypt. Egypt J. Bot. 53, 1-21.

Kadereit, J.W. 1993. A revision of Papaver sect. Meconidium. Edinburgh J. Bot. 50, 125-148. https://doi.org/10.1017/S0960428600002 523

Kadereit, J.W., Schwarzbach, A.E. and 
Jork, K.B. 1997. the phylogeny ofPapaver s. l. (Papaveraceae): Polyphyly or monophyly? Plant Syst. Evol. 204, 7598.

https://doi.org/https://doi.org/10.1007/BF 00982533

Keith, D. 1988. Floristic lists of New South Wales (III). Cunninghamia 2, 39-37.

Khedr, A. 2007. Assessment, classification, and analysis of microhabitats supporting globally significant Plant species: Conservation and Sustainable Use of Medicinal Plants in Arid and Semi-arid Eco-systems Project, Egypt. (Unpublished).

Kidron, G.J. and Zohar, M. 2010. Spatial evaporation patterns within a small drainage basin in the Negev Desert. J. Hydrol. 380, 376-385. https://doi.org/10.1016/j.jhydrol.2009.11. 012

Lomolino, M. V. 2001. Elevation gradients of species-density: Historical and prospective views. Glob. Ecol. Biogeogr. 10, 3-13. https://doi.org/10.1046/j.1466822x.2001.00229.x

Moustafa, A., El-Wahab, R., M, Z. and El-Rayes, A. 1998. Botanical survey of Saint Katherine Protectorate.

Moustafa, A. and Zaghloul, M. 1996. Environment and vegetation in the montane Saint Catherine area, south Sinai, Egypt 331-349.

Omar, K., Mohammed, A., Nagi, A., Elgamal, I., Elmarakby, A., Shalouf, A., Elasayed, A. and Mehana, S. 2015. Conservation Challenges inside Protected Areas of Egypt - Part II - St. Katherine Protectorate: Conservation Status Assessment of some threatened plant species.
POWO 2021. Plants of the World Online. Facilitated by the Royal Botanic Gardens, Kew. Published on the Internet; http://www.plantsoftheworldonline.org/ Retrieved 29 July 2021.

Rabei, S. Khedr, A. and El-Gamal, I. 2019. Clinopodium serpyllifolium subsp. barbatum (Lamiaceae) and Datura ferox (Solanaceae): New Records For The Flora of Egypt. Egypt. J. Bot. Vol. 59, No.1, pp. 233 - 239

Rafay, M., Ahmad Khan, R., Yaqoob, S. and Ahmad, M. 2013. Nutritional evaluation of major range grasses from Cholistan desert. Pakistan J. Nutr. 12, 23-29.

https://doi.org/10.3923/pjn.2013.23.29

Raunkiaer, C. 1934. The Plant Life Forms and Statistical Plant Geography. Clarendon Press, Oxford.

Sariyar, G. 2002. Biodiversity in the alkaloids of annual Papaver species of Turkish origin. Acta Pharm. Turc. 44, 159-168.

Shaltout, K., Heneidy, S., Al-Sodany, Y., Marie, A., Eid, E., Hatim, M. and ElGharaib, A. 2004. Floristic Survey of the Mountainous Southern Sinai: Saint Katherine Protectorate "B", Conservation and Sustainable Use of Medicinal Plants in Arid and Semi-arid Eco-systems.

Täckholm, V. 1974. Students' Flora of Egypt, 2nd ed. Cairo University, Cairo.

Walter, K.S. Harriet, G. 1997. IUCN Redlist.

Zahran, M. and Willis, A. 2009. The Vegetation of Egypt, 2nd ed. Springer.

Zohary, M. 1966, 1973 Flora Palaestina, part1- 2. The Israel Academy of Science and Humanities. 


\section{Floristic study of Saint Katherine protectorate, Sinai}

Appendix (1) the checklist of recorded taxa, $\mathrm{He}=$ Hemicryptophyte, $\mathrm{Ch}=$ chamaephyte, $\mathrm{Th}=$ Therophytes, $\mathrm{Ph}=$ Phaneroohyte, $\mathrm{Ge}=$ Geophyte, $\mathrm{Pa}=$ Parasite and $\mathrm{He}=$ Helophyte. $\mathrm{ME}=$ Mediterranean, $\mathrm{IT}=$ Irano- turanian, $\mathrm{SA}=$ Saharo - Arabian, SU = Sudanin and Sub T = sub trobic. $\dagger=$ recorded as herbarium specimen only

\begin{tabular}{|c|c|c|c|c|c|c|}
\hline Family & Accepted plant name & $\begin{array}{l}\text { life form } \\
\text { (Raunkiaer) }\end{array}$ & life spans & duration & Chorotype & $\begin{array}{c}\text { No. of } \\
\text { taxa }\end{array}$ \\
\hline Acanthaceae & BlePhris attenuata Napper & $\mathrm{Ch}$ & Perennial & Herb & IT,SA & 1 \\
\hline Aizoaceae & Aizoon canariense L. & Th & Annual & Herb & ME,SA,IT & 1 \\
\hline \multirow[t]{19}{*}{ Amaranthaceae } & $\begin{array}{l}\text { Aerva javanica (Burm. f.) Juss. ex } \\
\text { Schult. }\end{array}$ & $\mathrm{Ch}$ & Perennial & shrub & SA & 19 \\
\hline & $\begin{array}{l}\text { Agathophora alopecuroides (Delile) } \\
\text { Fenzlex }\end{array}$ & $\mathrm{Ch}$ & Perennial & $\begin{array}{l}\text { sub } \\
\text { Shrub }\end{array}$ & SA & \\
\hline & Amaranthus graecizans L. $\dagger$ & Th & Annual & Herb & pluri-regional & \\
\hline & Amaranthus hybridus L. $\dagger$ & Th & Annual & Herb & pluri-regional & \\
\hline & $\begin{array}{l}\text { Amaranthus blitum subsp. oleraceus (L.) } \\
\text { Costea }\end{array}$ & Th & Annual & Herb & pluri-regional & \\
\hline & Anabasis articulata (Forssk.) Moq. & $\mathrm{Ch}$ & Perennial & shrub & SA & \\
\hline & Anabasis setifera Moq. & $\mathrm{Ch}$ & Perennial & shrub & SA,IT & \\
\hline & Atriplex halimus L. & $\mathrm{Ph}$ & Perennial & shrub & ME,SA & \\
\hline & Atriplex turcomanica (Moq.) Boiss. & $\mathrm{Ch}$ & Perennial & shrub & IT & \\
\hline & Bassia arabica (Boiss.) Maire \& Weiller & Ch & Perennial & $\begin{array}{l}\text { sub } \\
\text { Shrub }\end{array}$ & SA & \\
\hline & Bassia eriophora (Schrad.) Asch. & $\mathrm{Ch}$ & Annual & Herb & SA,SU & \\
\hline & Bassia muricata (L.) Asch. & Th & Annual & Herb & SA,IT & \\
\hline & Beta vulgaris $\mathrm{L}$. & Th & $\begin{array}{l}\text { Annual / } \\
\text { perennial }\end{array}$ & Herb & ME,IT & \\
\hline & Chenopodium album L. & Th & Annual & Herb & pluri-regional & \\
\hline & $\begin{array}{l}\text { Chenopodiastrum murale (L.) S.Fuentes, } \\
\text { Uotila \& Borsch }\end{array}$ & Th & Annual & Herb & pluri-regional & \\
\hline & $\begin{array}{l}\text { Haloxylon salicornicum (Moq.) Bunge } \\
\text { ex Boiss. }\end{array}$ & $\mathrm{Ch}$ & Perennial & shrub & SA,IT & \\
\hline & Caroxylon imbricatum (Forssk.) Moq. & Ch & Perennial & shrub & SA & \\
\hline & Suaeda aegyptiaca (Hasselq.) Zohary & $\mathrm{He}$ & Perennial & Herb & SA & \\
\hline & Suaeda monoica Forssk. ex J.F. Gmel. & $\mathrm{Ph}$ & Perennial & shrub & SA,SU & \\
\hline Anacardiaceae & Pistacia khinjuk Stocks & $\mathrm{Ph}$ & Perennial & tree & IT & 1 \\
\hline \multirow[t]{7}{*}{ Apiaceae } & Deverra tortuosa (Desf.)DC. & $\mathrm{Ch}$ & Perennial & shrub & SA & \\
\hline & Deverra triradiata Hochst. ex Boiss. & Ch & Perennial & shrub & SA & 7 \\
\hline & Ferula Sinaica Boiss. & $\mathrm{He}$ & Perennial & Herb & IT & \\
\hline & Foeniculum vulgare Mill. & $\mathrm{He}$ & Perennial & Herb & ME,IT & \\
\hline & Pycnocycla tomentosa Decne. & $\mathrm{He}$ & Perennial & Herb & SA & \\
\hline & Scandix stellata Banks \& Sol. & Th & Annual & Herb & IT & \\
\hline & Zosima absinthifolia (Vent.) Link. & $\mathrm{He}$ & Perennial & Herb & IT & \\
\hline \multirow[t]{8}{*}{ Apocynaceae } & Calotropis procera (Aiton) W.T. Aiton. & $\mathrm{Ph}$ & Perennial & shrub & SA & 7 \\
\hline & Caudanthera Sinaica (Decne.) Plowes & $\mathrm{He}$ & Perennial & Herb & SA & \\
\hline & Cynanchum acutum L. & $\mathrm{Ch}$ & Perennial & Herb & SA & \\
\hline & Gomphocarpus Sinaicus Boiss. & $\mathrm{Ch}$ & Perennial & shrub & SA & \\
\hline & $\begin{array}{l}\text { Leptadenia pyrotechnica (Forssk.) } \\
\text { Decne. }\end{array}$ & $\mathrm{Ph}$ & Perennial & shrub & SA & \\
\hline & Pergularia tomentosa L. & Ch & Perennial & shrub & SA & \\
\hline & Periploca aphylla subsp. Aphylla & $\mathrm{Ph}$ & Perennial & shrub & SU & \\
\hline & $\begin{array}{l}\text { Solenostemma oleifolium (Nectoux) } \\
\text { Bullock \& E.A. Bruce ex Maire }\end{array}$ & $\mathrm{Ph}$ & Perennial & shrub & SA & \\
\hline
\end{tabular}




\section{Rabei et al.}

\begin{tabular}{|c|c|c|c|c|c|c|}
\hline Family & Accepted plant name & $\begin{array}{l}\text { life form } \\
\text { (Raunkiaer) }\end{array}$ & life spans & duration & Chorotype & $\begin{array}{c}\text { No. of } \\
\text { taxa }\end{array}$ \\
\hline Arecaceae & Phoenix dactylifera $\mathrm{L}$. & $\mathrm{Ch}$ & Perennial & tree & SA & 1 \\
\hline Asparagaceae & Asparagus horridus L. & $\mathrm{Ge}$ & Perennial & shrub & ME,SA & 1 \\
\hline Asphodelaceae & Asphodelus tenuifolius Cav. & Th & Annual & Herb & ME,SA,IT & 1 \\
\hline \multirow[t]{37}{*}{ Asteraceae } & $\begin{array}{l}\text { Achillea fragrantissima (Forssk.) Sch. } \\
\text { Bip. }\end{array}$ & $\mathrm{He}$ & Perennial & shrub & IT,SA & 63 \\
\hline & Achillea tenuifolia Lam. & $\mathrm{He}$ & Perennial & Herb & IT & \\
\hline & AntHeis melampodina Delile $\dagger$ & Th & Annual & Herb & SA,IT & \\
\hline & Artemisia herba-alba Asso & Ch & Perennial & shrub & IT & \\
\hline & Artemisia judaica L. & $\mathrm{Ch}$ & Perennial & shrub & SA & \\
\hline & Asteriscus graveolens (Forssk.) Less. & $\mathrm{He}$ & Perennial & shrub & SA & \\
\hline & Atractylis carduus (Forssk.) C. Chr. & Ch & Perennial & $\begin{array}{l}\text { sub } \\
\text { Shrub }\end{array}$ & SA & \\
\hline & $\begin{array}{l}\text { Atractylis mernephthae Asch. \& } \\
\text { Schweinf. \& Letourn. }\end{array}$ & Th & Annual & Herb & SA & \\
\hline & Bidens pilosa L. & Th & Annual & Herb & ME,IT,EU & \\
\hline & Blumea bovei (DC.) Vatke & $\mathrm{He}$ & Perennial & Herb & SA & \\
\hline & Brocchia cinerea (Delile) Vis. & Th & Annual & Herb & SA & \\
\hline & Calendula arvensis L. & Th & Annual & Herb & ME,IT & \\
\hline & Carduus getulus Pomel. & Th & Annual & Herb & SA & \\
\hline & Carthamus nitidus Boiss. & Th & Annual & Herb & SA & \\
\hline & Centaurea eryngioides Lam. & $\mathrm{He}$ & Perennial & Herb & SA,IT & \\
\hline & Centaurea scoparia Sieber ex Spreng. & Ch & Perennial & $\begin{array}{l}\text { sub } \\
\text { Shrub }\end{array}$ & SA & \\
\hline & Centaurea Sinaica DC. & Th & Annual & Herb & SA & \\
\hline & Chiliadenus montanus (Vahl) Brullo. & $\mathrm{He}$ & Perennial & shrub & SA & \\
\hline & Cichorium pumilum Jacq. & Th & Annual & Herb & ME,IT & \\
\hline & Crepis sancta (L.) Bornm. & Th & Annual & Herb & ME,SA,IT & \\
\hline & Echinops glaberrimus DC. & $\mathrm{He}$ & Perennial & Herb & SA & \\
\hline & Erigeron bonariensis L. & Th & Annual & Herb & SU & \\
\hline & Eschenbachia stricta (Willd.) Raizada & Ch & Perennial & Herb & SA & \\
\hline & Filago desertorum Pomel & Th & Annual & Herb & SA,IT & \\
\hline & Glebionis coronaria (L.) Cass. ex Spach & Th & Annual & Herb & $\mathrm{ME}$ & \\
\hline & Gymnarrhena micrantha Desf. $\dagger$ & Th & Annual & Herb & SA,IT & \\
\hline & Ifloga spicata subsp. albescens Chrtek & Th & Annual & Herb & ME,SA,IT & \\
\hline & Ifloga spicata subsp. spicata & Th & Annual & Herb & ME,SA,IT & \\
\hline & $\begin{array}{l}\text { Iphiona mucronata (Forssk.) Asch. \& } \\
\text { Schweinf. }\end{array}$ & Ch & Perennial & shrub & SA & \\
\hline & Iphiona scabra DC. ex Decne. & Ch & Perennial & shrub & SA & \\
\hline & Koelpinia linearis Pall. & Th & Annual & Herb & ME,SA,IT & \\
\hline & Lactuca orientalis (Boiss.) Boiss. & Ch & Perennial & shrub & IT & \\
\hline & Lactuca saligna L. & Th & $\begin{array}{l}\text { Annual/ } \\
\text { bienniaal }\end{array}$ & Herb & ME,SA,IT & \\
\hline & Lactuca serriola L. & $\mathrm{Ge}$ & $\begin{array}{l}\text { Annual/ } \\
\text { bienniaal }\end{array}$ & Herb & ME,IT & \\
\hline & Lactuca undulata Ledeb. & Th & Annual & Herb & IT & \\
\hline & Lasiopogon muscoides (Desf.) DC. & Th & Annual & Herb & SA & \\
\hline & Launaea capitata (Spreng.) Dandy & Th & Annual & Herb & SA & \\
\hline
\end{tabular}




\section{Floristic study of Saint Katherine protectorate, Sinai}

\begin{tabular}{|c|c|c|c|c|c|c|}
\hline Family & Accepted plant name & $\begin{array}{l}\text { life form } \\
\text { (Raunkiaer) }\end{array}$ & life spans & duration & Chorotype & $\begin{array}{c}\text { No. of } \\
\text { taxa }\end{array}$ \\
\hline & Launaea mucronata (Forssk.) Muschl. & $\mathrm{He}$ & Perennial & Herb & SA & \\
\hline & Launaea nudicaulis (L.) Hook. f. & $\mathrm{He}$ & Perennial & Herb & SA,IT & \\
\hline & $\begin{array}{l}\text { Launaea spinosa (Forssk.) Sch. Bip. Ex } \\
\text { Kuntze }\end{array}$ & $\mathrm{Ch}$ & Perennial & shrub & SA & \\
\hline & Leysera leyseroides (Desf.) Maire & Th & Annual & Herb & SA,SU & \\
\hline & $\begin{array}{l}\text { Nidorella aegyptiaca (L.) J.C.Manning } \\
\text { \& Goldblatt }\end{array}$ & Th & Annual & Herb & SU & \\
\hline & Onopordum ambiguum Fresen. & He & Biennial & Herb & IT,SA & \\
\hline & Osteospermum vaillantii (Decne.) Norl & $\mathrm{Ch}$ & Perennial & Herb & SA,SU & \\
\hline & Pallenis hierochuntica (Michon) Greuter & Th & Annual & Herb & SA & \\
\hline & Pallenis spinosa (L.) Cass. & $\mathrm{He}$ & $\begin{array}{l}\text { Annual / } \\
\text { perennial }\end{array}$ & Herb & ME & \\
\hline & $\begin{array}{l}\text { Phgnalon barbeyanum Asch. } \\
\text { \&Schweinf }\end{array}$ & $\mathrm{Ch}$ & Perennial & shrub & SA & \\
\hline & Phgnalon nitidum Fresen. & $\mathrm{Ch}$ & Perennial & Herb & IT & \\
\hline & Phgnalon Sinaicum Bornm. \& Kneuck. & $\mathrm{Ch}$ & Perennial & shrub & IT & \\
\hline & Picris cyanocarpa Boiss. & Th & Annual & Herb & SA & \\
\hline & Pulicaria incisa (Lam.) DC. & $\mathrm{Ch}$ & Perennial & Herb & SA,SU & \\
\hline & Pulicaria inuloides DC. & $\mathrm{He}$ & Perennial & Herb & SA & \\
\hline & Pulicaria undulata (L.) C. A. Mey. & $\mathrm{Ch}$ & Perennial & shrub & SA,SU & \\
\hline & ReiChrdia tingitana (L.) Roth. & Th & Annual & Herb & ME,SA,IT & \\
\hline & Scorzonera schweinforthii Boiss. & $\mathrm{He}$ & Perennial & Herb & SA & \\
\hline & Senecio flavus (Decne.) Sch. Bip. & Th & Annual & Herb & SA & \\
\hline & Senecio glaucus L. & Th & Annual & Herb & ME,SA,IT & \\
\hline & Sonchus asper (L.) Hill & Th & Annual & Herb & ME & \\
\hline & Sonchus oleraceus L. & Th & Annual & Herb & ME & \\
\hline & $\begin{array}{l}\text { Tanacetum sinaicum (Fresen.) Delile ex } \\
\text { K.Bremer \& Humphries }\end{array}$ & $\mathrm{Ch}$ & Perennial & shrub & IT & \\
\hline & $\begin{array}{l}\text { Tragopogon porrifolius subsp. } \\
\text { porrifolius }\end{array}$ & $\mathrm{Ch}$ & $\begin{array}{l}\text { Biennial/ } \\
\text { Perennial }\end{array}$ & Herb & ME & \\
\hline & $\begin{array}{l}\text { Urospermum picroides (L.) F.W. } \\
\text { Schmidt. }\end{array}$ & Th & Annual & Herb & ME,SA,IT & \\
\hline & Zoegia purpurea Fresen. & Th & Annual & Herb & SA,IT & \\
\hline \multirow[t]{12}{*}{ Boraginaceae } & Alkanna orientalis (L.) Boiss. & $\mathrm{Ch}$ & Perennial & Herb & ME,IT & 14 \\
\hline & Gastrocotyle hispida (Forssk.) Bunge & Th & Annual & Herb & SA,IT & \\
\hline & Anchusa milleri Lam. ex Spreng. & Th & Annual & Herb & IT,SA & \\
\hline & $\begin{array}{l}\text { Arnebia decumbens (Vent.) Coss. \& } \\
\text { Kralik } \dagger\end{array}$ & Th & Annual & Herb & SA,IT & \\
\hline & Echium horridum Batt. & Th & $\begin{array}{l}\text { Annual / } \\
\text { perennial }\end{array}$ & Herb & ME,SA & \\
\hline & Heliotropium arbainense Fresen. & $\mathrm{Ch}$ & Perennial & $\begin{array}{l}\text { sub } \\
\text { Shrub }\end{array}$ & SA & \\
\hline & Heliotropium bacciferum Forssk. & $\mathrm{Ch}$ & Perennial & Herb & SA & \\
\hline & $\begin{array}{l}\text { Heliotropium digynum (Forssk.) Asch. } \\
\text { ex C.Chr. }\end{array}$ & $\mathrm{Ch}$ & Perennial & Herb & SA & \\
\hline & $\begin{array}{l}\text { Pseudolappula Sinaica (A.DC.) } \\
\text { Khoshsokhan, Sherafati \& Kaz.Osaloo }\end{array}$ & Th & Annual & Herb & SA & \\
\hline & $\begin{array}{l}\text { Lappula spinocarpos (Forssk.) Asch. ex } \\
\text { Kunze. }\end{array}$ & Th & Annual & Herb & SA,IT & \\
\hline & $\begin{array}{l}\text { Microparacaryum intermedium (Fresen.) } \\
\text { Hilger \& Podlech }\end{array}$ & Th & Annual & Herb & SA,IT & \\
\hline & Paracaryum rugulosum (DC.) Bosis. & $\mathrm{He}$ & Biennial & Herb & IT,SA & \\
\hline
\end{tabular}




\section{Rabei et al.}

\begin{tabular}{|c|c|c|c|c|c|c|}
\hline Family & Accepted plant name & $\begin{array}{l}\text { life form } \\
\text { (Raunkiaer) }\end{array}$ & life spans & duration & Chorotype & $\begin{array}{c}\text { No. of } \\
\text { taxa }\end{array}$ \\
\hline & Trichodesma africanum (L.) Sm. & Th & Annual & Herb & SA & \\
\hline & Trichodesma ehrenbergii Schweinf. & He & $\begin{array}{l}\text { Annual/ } \\
\text { bienniaal }\end{array}$ & Herb & SU & \\
\hline \multirow[t]{27}{*}{ Brassicaceae } & Arabis recta Vill. & Th & Annual & Herb & IT & 27 \\
\hline & Capsella bursa-pastoris (L.) Medik & Th & Annual & Herb & pluri-regional & \\
\hline & Carrichtera annua (L.) DC. & Th & Annual & Herb & SA & \\
\hline & Clypeola jonthlaspi L. & Th & Annual & Herb & ME,IT & \\
\hline & $\begin{array}{l}\text { Cuprella homalocarpa (Fisch. \& } \\
\text { C.A.Mey.) Salmerón-Sánchez, Mota \& } \\
\text { Fuertes }\end{array}$ & Th & Annual & Herb & IT & \\
\hline & Diplotaxis acris (Forssk.) Boiss. & Th & Annual & Herb & SA & \\
\hline & Diplotaxis harra (Forssk.) Boiss. & $\mathrm{Ch}$ & Perennial & Herb & SA & \\
\hline & Eruca vesicaria (L.) Cav. & Th & Annual & Herb & ME & \\
\hline & Farsetia aegyptia Turra & $\mathrm{Ch}$ & Perennial & shrub & SU & \\
\hline & Farsetia longisiliqua Decne. & $\mathrm{Ch}$ & Perennial & Herb & SU & \\
\hline & Hornungia procumbens (L.) Hayek & Th & Annual & Herb & ME,SA,IT & \\
\hline & Isatis microcarpa J.Gay ex Boiss. & Th & Annual & Herb & SA & \\
\hline & $\begin{array}{l}\text { Lobularia canariensis subsp. marginata } \\
\text { (Webb ex Coss.) L.Borgen } †\end{array}$ & Th & Annual & Herb & IT & \\
\hline & lepidium draba $\mathrm{L}$. & Th & Annual & Herb & ME,IT & \\
\hline & $\begin{array}{l}\text { Malcolmia africana (L.) R. Br. in W. T. } \\
\text { Aiton } \dagger\end{array}$ & Th & Annual & Herb & SA,IT & \\
\hline & Matthiola arabica Boiss. & He & Perennial & Herb & SA & \\
\hline & Matthiola longipetala (Vent.) DC. & Th & Annual & Herb & ME,IT & \\
\hline & Morettia canescens Boiss. & $\mathrm{Ch}$ & Perennial & Herb & ME & \\
\hline & Moricandia Sinaica (Boiss.) Boiss. & $\mathrm{Ch}$ & Perennial & Herb & SA & \\
\hline & RaPhnus raPhnistrum L. & Th & Annual & Herb & ME,EU & \\
\hline & Savignya parviflora (Delile) Webb & Th & Annual & Herb & SA & \\
\hline & Schimpera arabica Hochst. \& Steud. & Th & Annual & Herb & SA & \\
\hline & Schouwia purpurea (Forssk.) Schweinf. & Th & Annual & Herb & SA & \\
\hline & Sisymbrium erysimoides Desf. & Th & Annual & Herb & ME,IT & \\
\hline & Sisymbrium irio L. & Th & Annual & Herb & ME,IT & \\
\hline & Sisymbrium septulatum DC. & Th & Annual & Herb & IT & \\
\hline & Zilla spinosa (L.) Prrantl & $\mathrm{Ch}$ & Perennial & shrub & SA & \\
\hline \multirow[t]{2}{*}{ Capparaceae } & Capparis cartilaginea Decne & $\mathrm{Ph}$ & Perennial & shrub & SA & 2 \\
\hline & Capparis spinosa L. & $\mathrm{Ch}$ & Perennial & shrub & ME,SA,IT & \\
\hline \multirow[t]{2}{*}{ Caprifoliaceae } & PterocePhlus arabicus Boiss. & $\mathrm{Ch}$ & Perennial & shrub & $\begin{array}{l}\text { Endemic \& } \\
\text { Steno-endemic }\end{array}$ & 2 \\
\hline & PterocePhlus sanctus Decne. & $\mathrm{Ch}$ & Perennial & shrub & IT & \\
\hline \multirow[t]{6}{*}{ Caryophyllaceae } & Arenaria deflexa Decne. & $\mathrm{Ch}$ & Perennial & Herb & ME & 25 \\
\hline & Bolanthus hirsutus (Labill.) Barkoudah & He & Perennial & Herb & ME & \\
\hline & Bufonia multiceps Decne. & He & Perennial & Herb & $\begin{array}{l}\text { Endemic \& } \\
\text { Steno-endemic }\end{array}$ & \\
\hline & Cerastium dichotomum L. $\dagger$ & Th & Annual & Herb & ME,IT & \\
\hline & Dianthus libanotis Labill. & $\mathrm{Ch}$ & Perennial & Herb & ME,IT & \\
\hline & Dianthus Sinaicus Boiss. & $\mathrm{Ch}$ & Perennial & Herb & IT & \\
\hline
\end{tabular}




\section{Floristic study of Saint Katherine protectorate, Sinai}

\begin{tabular}{|c|c|c|c|c|c|c|}
\hline Family & Accepted plant name & $\begin{array}{l}\text { life form } \\
\text { (Raunkiaer) }\end{array}$ & life spans & duration & Chorotype & $\begin{array}{c}\text { No. of } \\
\text { taxa }\end{array}$ \\
\hline & Gymnocarpos decander Forssk. & $\mathrm{Ch}$ & Perennial & shrub & SA & \\
\hline & Gypsophila capillaris (Forssk.) C. Chr. & Ch & Perennial & Herb & IT & \\
\hline & Gypsophila viscosa Murray & Th & Annual & Herb & IT & \\
\hline & $\begin{array}{l}\text { Eremogone picta (Sm.) Dillenb. \& } \\
\text { Kadereit }\end{array}$ & Th & Annual & Herb & IT & \\
\hline & Paronychia Sinaica Fresen. & $\mathrm{He}$ & Perennial & Herb & IT & \\
\hline & $\begin{array}{l}\text { Polycarpaea repens (Forssk.) Asch. \& } \\
\text { Schweinf. }\end{array}$ & Th & Annual & Herb & SA,SU & \\
\hline & $\begin{array}{l}\text { Polycarpaea robbairea (Kuntze) } \\
\text { Greuter \& Burdet }\end{array}$ & Th & Annual & Herb & SA & \\
\hline & Pteranthus dichotomus Forssk. & Th & Annual & Herb & SA & \\
\hline & $\begin{array}{l}\text { Gymnocarpos sclerocePhlus (Decne.) } \\
\text { Dahlgren \& Thulin }\end{array}$ & Th & Annual & Herb & SA & \\
\hline & Silene arabica Boiss. & Th & Annual & Herb & ME,IT & \\
\hline & Silene leucophylla Boiss. & $\mathrm{He}$ & Perennial & Herb & $\begin{array}{l}\text { Endemic \& } \\
\text { Steno-endemic }\end{array}$ & \\
\hline & Silene linearis Decne. & Th & Annual & Herb & SU & \\
\hline & $\begin{array}{l}\text { Silene odontopetala subsp. congesta } \\
\text { (Boiss.) Melzh. }\end{array}$ & He & Perennial & Herb & $\mathrm{ME}$ & \\
\hline & Silene oreoSinaica Chowdhuri & $\mathrm{He}$ & Perennial & Herb & $\begin{array}{l}\text { Endemic \& } \\
\text { Steno-endemic }\end{array}$ & \\
\hline & Silene schimperiana Boiss. & Не & Perennial & Herb & $\begin{array}{l}\text { Endemic \& } \\
\text { Steno-endemic }\end{array}$ & \\
\hline & Silene villosa Forssk. & Th & Annual & Herb & SA & \\
\hline & $\begin{array}{l}\text { Spergularia flaccida (Madden) } \\
\text { I.M.Turner }\end{array}$ & Th & Annual & Herb & ME,SA,IT & \\
\hline & Spergularia diandra (Guss.) Heldr. & Th & Annual & Herb & SA & \\
\hline & Spergularia marina (L.) Besser & Не & $\begin{array}{l}\text { Annual / } \\
\text { perennial }\end{array}$ & Herb & ME,IT & \\
\hline & Stellaria media (L.)Vill. & Th & Annual & Herb & pluri-regional & \\
\hline \multirow[t]{4}{*}{ Cistaceae } & HeliantHeum aegyptiacum (L.) Mill. & Th & Annual & Herb & ME,IT & 4 \\
\hline & HeliantHeum kahiricum Delile & Ch & Perennial & shrub & SA & \\
\hline & HeliantHeum lippii (L.) Dum. Cours. & Ch & Perennial & shrub & SA & \\
\hline & $\begin{array}{l}\text { HeliantHeum sancti-antonii Schweinf. } \\
\text { ex Boiss. }\end{array}$ & Ch & Perennial & shrub & SA & \\
\hline \multirow[t]{4}{*}{ Cleomaceae } & Cleome amblyocarpa Barratte \& Murb. & Th & Annual & Herb & SA,SU & 4 \\
\hline & Cleome arabica L. & Ch & Perennial & shrub & SU & \\
\hline & Cleome chrysantha Decne. & $\mathrm{Ch}$ & Perennial & Herb & SA,SU & \\
\hline & Cleome droserifolia (Forssk.) Delile & Ch & Perennial & shrub & SU & \\
\hline \multirow[t]{2}{*}{ Colchicaceae } & Colchicum schimperi Janka ex Stef. & $\mathrm{Ge}$ & Perennial & Herb & SA & 2 \\
\hline & Colchicum ritchii R.Br. & $\mathrm{Ge}$ & Perennial & Herb & SA & \\
\hline \multirow[t]{2}{*}{ Convolvulaceae } & Convolvulus arvensis L. & $\mathrm{Ge}$ & Annual & Climber & pluri-regional & 2 \\
\hline & Cuscuta planiflora Ten. & $\mathrm{Pa}$ & Annual & Climber & ME,SA & \\
\hline Crassulaceae & Umbilicus horizontalis (Guss.) DC. & $\mathrm{Ge}$ & Perennial & Herb & $\mathrm{ME}$ & 1 \\
\hline \multirow[t]{2}{*}{ Cucurbitaceae } & Citrullus colocynthis (L.) Schrad. & $\mathrm{He}$ & Perennial & Climber & ME,SA,IT & 2 \\
\hline & Cucumis prophetarum L. & $\mathrm{He}$ & Annual & Climber & SA & \\
\hline \multirow[t]{4}{*}{ Сyperaceae } & Cyperus laevigatus L. & $\mathrm{He}$ & Perennial & Herb & ME,IT & 4 \\
\hline & Cyperus rotundus $\mathrm{L}$. & $\mathrm{Ge}$ & Perennial & Herb & pluri-regional & \\
\hline & Schoenus nigricans L. & На & Perennial & Herb & ME,IT,EU & \\
\hline & Scirpoides holoschoenus (L.) Soják & $\mathrm{He}$ & Perennial & Herb & ME,SA,IT & \\
\hline
\end{tabular}


Rabei et al.

\begin{tabular}{|c|c|c|c|c|c|c|}
\hline Family & Accepted plant name & $\begin{array}{l}\text { life form } \\
\text { (Raunkiaer) }\end{array}$ & life spans & duration & Chorotype & $\begin{array}{c}\text { No. of } \\
\text { taxa }\end{array}$ \\
\hline \multirow[t]{3}{*}{ Ephedraceae } & Ephedra alata Decne. & $\mathrm{Ch}$ & Perennial & shrub & ME,SA & 3 \\
\hline & Ephedra foliata Boiss. ex C.A. Mey. & $\mathrm{Ph}$ & Perennial & shrub & SU & \\
\hline & $\begin{array}{l}\text { Ephedra pachyclada subsp. sinaica } \\
\text { (Riedl) Freitag \& Maier-St. }\end{array}$ & $\mathrm{Ph}$ & Perennial & shrub & IT & \\
\hline Equisetaceae & Equisetum ramosissimum Desf. & He & Perennial & Herb & pluri-regional & 1 \\
\hline \multirow[t]{9}{*}{ Euphorbiaceae } & $\begin{array}{l}\text { Chrozophora oblongifolia (Delile) } \\
\text { A.Juss. ex Spreng. }\end{array}$ & $\mathrm{Ch}$ & Perennial & Herb & SU & 9 \\
\hline & Euphorbia Chmaepeplus Boiss. \& Gaill. & Th & Annual & Herb & SA,IT & \\
\hline & Euphorbia granulata Forssk. & Th & Annual & Herb & SA & \\
\hline & Euphorbia helioscopia L. & Th & Annual & Herb & ME & \\
\hline & Euphorbia hirta L. & Th & Annual & Herb & pluri-regional & \\
\hline & Euphorbia obovata Decne. & He & Perennial & sub Shrub & $\begin{array}{l}\text { Endemic \& } \\
\text { Steno-endemic }\end{array}$ & \\
\hline & Euphorbia peplus L. & Th & Annual & Herb & ME,IT,EU & \\
\hline & Euphorbia prostrata Aiton. & Th & Annual & Herb & pluri-regional & \\
\hline & Euphorbia retusa Forssk. & He & Perennial & Herb & SA & \\
\hline \multirow[t]{26}{*}{ Fabaceae } & Alhagi graecorum Boiss. & He & Perennial & shrub & ME,IT & 34 \\
\hline & $\begin{array}{l}\text { Argyrolobium arabicum (Decne.) Jaub. } \\
\text { \& Spach }\end{array}$ & Th & $\begin{array}{l}\text { Annual / } \\
\text { perennial }\end{array}$ & Herb & SA & \\
\hline & Astragalus amalecitanus Boiss. & $\mathrm{Ch}$ & Perennial & Herb & IT & \\
\hline & Astragalus asterias Steven & Th & Annual & Herb & ME,SA & \\
\hline & Astragalus bombycinus Boiss. & Th & Annual & Herb & SA & \\
\hline & Astragalus caprinus L. & He & Perennial & Herb & SA & \\
\hline & Astragalus crenatus Schult. & Th & Annual & Herb & SA,IT & \\
\hline & Astragalus cretaceus Boiss. \& Kotschy & He & Perennial & Herb & IT & \\
\hline & $\begin{array}{l}\text { Astragalus dactylocarpus subsp. } \\
\text { acinacifer (Boiss.) E.Ott }\end{array}$ & $\mathrm{He}$ & Perennial & sub Shrub & SA & \\
\hline & Astragalus echinus DC. & $\mathrm{Ch}$ & Perennial & shrub & ME,IT & \\
\hline & Astragalus eremophilus Boiss. & Th & Annual & Herb & SA & \\
\hline & Astragalus fruticosus Forssk. $\dagger$ & He & Perennial & $\begin{array}{l}\text { Herb/ } \\
\text { Subshrub }\end{array}$ & SA & \\
\hline & Astragalus palaestinus Eig. & $\mathrm{He}$ & $\begin{array}{l}\text { Annual / } \\
\text { perennial }\end{array}$ & Herb & ME,IT & \\
\hline & Astragalus sieberi DC. & $\mathrm{Ch}$ & Perennial & sub Shrub & SA & \\
\hline & Astragalus spinosus (Forssk.) Muschl. & $\mathrm{Ch}$ & Perennial & shrub & IT & \\
\hline & Astragalus tribuloides Delile & Th & Annual & Herb & IT,SA & \\
\hline & Bituminaria flaccida (Nábelek) Greuter & He & Perennial & Herb & $\mathrm{ME}$ & \\
\hline & Colutea istria Mill. & $\mathrm{Ph}$ & Perennial & shrub & IT & \\
\hline & Crotalaria aegyptiaca Benth. & He & Perennial & shrub & SA & \\
\hline & $\begin{array}{l}\text { Leobordea platycarpa (Viv.) B.-E.van } \\
\text { Wyk \& Boatwr. }\end{array}$ & $\mathrm{Ge}$ & $\begin{array}{l}\text { Annual / } \\
\text { perennial }\end{array}$ & Herb & SA,SU & \\
\hline & Lotus hebranicus Hochst. ex Brand & He & Perennial & sub Shrub & SA & \\
\hline & Medicago laciniata (L.) Mill. & Th & Annual & Herb & SA & \\
\hline & Medicago sativa $\mathrm{L}$. & He & Perennial & Herb & ME,IT & \\
\hline & Melilotus indicus (L.) All. & Th & Annual & Herb & $\mathrm{ME}$ & \\
\hline & Onobrychis ptolemaica (Delile) DC. & He & Perennial & Herb & SA & \\
\hline & Ononis sicula Guss. & Th & Annual & Herb & ME,SA,IT & \\
\hline
\end{tabular}




\section{Floristic study of Saint Katherine protectorate, Sinai}

\begin{tabular}{|c|c|c|c|c|c|c|}
\hline Family & Accepted plant name & $\begin{array}{l}\text { life form } \\
\text { (Raunkiaer) }\end{array}$ & life spans & duration & Chorotype & $\begin{array}{c}\text { No. of } \\
\text { taxa }\end{array}$ \\
\hline & $\begin{array}{l}\text { Retama raetam (Forssk.) Webb. \& } \\
\text { Berthel. }\end{array}$ & $\mathrm{Ph}$ & Perennial & shrub & SA & \\
\hline & Senna alexandrina Mill. & $\mathrm{He}$ & Perennial & shrub & SU & \\
\hline & Senna italica Mill. & $\mathrm{He}$ & Perennial & $\begin{array}{l}\text { sub } \\
\text { Shrub }\end{array}$ & SU & \\
\hline & $\begin{array}{l}\text { Tephrosia purpurea subsp. leptostachya } \\
\text { (DC.) Brummitt }\end{array}$ & Ch & Perennial & Herb & SA & \\
\hline & $\begin{array}{l}\text { Vachellia tortilis subsp. raddiana (Savi) } \\
\text { Kyal. \& Boatwr. }\end{array}$ & $\mathrm{Ph}$ & Perennial & tree & SA,SU & \\
\hline & Vachellia tortilis subsp. tortilis & $\mathrm{Ph}$ & Perennial & tree & SA,SU & \\
\hline & Vicia articulata Hornem & Th & Annual & Herb & $\mathrm{ME}$ & \\
\hline & Vicia monantha Retz. & Th & Annual & Herb & SA & \\
\hline Gentianaceae & $\begin{array}{l}\text { Centaurium pulchellum (Sw.) Hayek ex } \\
\text { Hand-Mazz., Stadlm., Janch. \& Faltis }\end{array}$ & Th & Annual & Herb & ME,IT & 1 \\
\hline \multirow[t]{4}{*}{ Geraniaceae } & Erodium cicutarium (L.) L'Her. & Th & Annual & Herb & ME,IT & 4 \\
\hline & Erodium crassifolium L'Her. & $\mathrm{He}$ & Perennial & Herb & SA & \\
\hline & Erodium laciniatum (Cav.) Willd. & Th & Annual & Herb & ME & \\
\hline & Geranium molle L. & Th & Annual & Herb & $\mathrm{ME}$ & \\
\hline Hypericaceae & Hypericum Sinaicum Hochst. ex Boiss & $\mathrm{Ch}$ & Perennial & Herb & IT & 1 \\
\hline \multirow[t]{4}{*}{ Juncaceae } & Juncus acutus L. & $\mathrm{He}$ & Perennial & Herb & ME,IT & 4 \\
\hline & Juncus bufonius L. & Th & Annual & Herb & ME,IT & \\
\hline & Juncus punctorius L.f. & $\mathrm{He}$ & Perennial & Herb & SA & \\
\hline & Juncus rigidus Desf. & $\mathrm{He}$ & Perennial & Herb & IT,SA & \\
\hline \multirow[t]{20}{*}{ Lamiaceae } & $\begin{array}{l}\text { Ajuga Chmaepitys subsp. tridactylites } \\
\text { (Ging. ex Benth.) P.H.Davis }\end{array}$ & $\mathrm{He}$ & Perennial & Herb & IT & 26 \\
\hline & Ballota kaiseri Täckh & $\mathrm{He}$ & Perennial & shrub & $\begin{array}{l}\text { Endemic \& } \\
\text { Steno-endemic }\end{array}$ & \\
\hline & Ballota saxatilis Sieber ex C.Presl & $\mathrm{He}$ & Perennial & shrub & ME,IT & \\
\hline & $\begin{array}{l}\text { Clinopodium barbatum (P.H.Davis) } \\
\text { Melnikov }\end{array}$ & Ch & Perennial & shrub & ME & \\
\hline & Lamium amplexicaule $\mathrm{L}$. & Th & Annual & Herb & ME,IT & \\
\hline & Lavandula coronopifolia Poir. & Ch & Perennial & shrub & SA,SU & \\
\hline & Lavandula pubescens Decne. & $\mathrm{He}$ & Perennial & shrub & ME,SA & \\
\hline & $\begin{array}{l}\text { Mentha longifolia var. schimperi (Briq.) } \\
\text { Briq. }\end{array}$ & $\mathrm{He}$ & Perennial & Herb & ME,IT & \\
\hline & Micromeria serbaliana Danin \& Hedge & $\mathrm{He}$ & Perennial & Herb & $\begin{array}{l}\text { Endemic \& } \\
\text { Steno-endemic }\end{array}$ & \\
\hline & Micromeria Sinaica Benth. & Ch & Perennial & shrub & SA & \\
\hline & Nepeta septemcrenata Ehrenb. ex Benth. & Ch & Perennial & shrub & ME,SA & \\
\hline & $\begin{array}{l}\text { Origanum syriacum subsp. sinaicum } \\
\text { (Boiss.) Greater \& Burdet }\end{array}$ & Ch & Perennial & shrub & $\begin{array}{l}\text { Endemic \& } \\
\text { Steno-endemic }\end{array}$ & \\
\hline & $\begin{array}{l}\text { Otostegia fruticosa subsp. schimperi } \\
\text { (Benth.) Sebald }\end{array}$ & Ch & Perennial & shrub & SA & \\
\hline & Phlomis aurea Decne. & $\mathrm{Ch}$ & Perennial & shrub & $\begin{array}{l}\text { Endemic \& } \\
\text { Steno-endemic }\end{array}$ & \\
\hline & $\begin{array}{l}\text { Pseudodictamnus undulatus (Benth.) } \\
\text { Salmaki \& Siadati }\end{array}$ & Ch & Perennial & shrub & ME & \\
\hline & Salvia aegyptiaca L. & Ch & Perennial & $\begin{array}{l}\text { sub } \\
\text { Shrub }\end{array}$ & SA,IT & \\
\hline & Salvia deserti Decne. & Не & Perennial & $\begin{array}{l}\text { sub } \\
\text { Shrub }\end{array}$ & SA & \\
\hline & Salvia lanigra Poir. & $\mathrm{Ch}$ & Perennial & Herb & ME,SA & \\
\hline & Salvia multicaulis Vahl & Ch & Perennial & shrub & IT & \\
\hline & Salvia sclarea L. & $\mathrm{He}$ & Perennial & Herb & ME,IT & \\
\hline
\end{tabular}




\section{Rabei et al.}

\begin{tabular}{|c|c|c|c|c|c|c|}
\hline Family & Accepted plant name & $\begin{array}{l}\text { life form } \\
\text { (Raunkiaer) }\end{array}$ & life spans & duration & Chorotype & $\begin{array}{c}\text { No. of } \\
\text { taxa }\end{array}$ \\
\hline & Salvia spinosa L. & $\mathrm{He}$ & Perennial & Herb & IT & \\
\hline & Stachys aegyptiaca Pers. & Ch & Perennial & shrub & ME,SA,IT & \\
\hline & Teucrium leucocladum Boiss. & $\mathrm{Ch}$ & Perennial & shrub & SA & \\
\hline & Teucrium polium L. & $\mathrm{Ch}$ & Perennial & shrub & ME,IT & \\
\hline & Thymus decussatus Benth. & $\mathrm{Ch}$ & Perennial & shrub & SA & \\
\hline & Ziziphora tenuior L. & Th & Annual & Herb & SA & \\
\hline \multirow[t]{5}{*}{ Malvaceae } & Abutilon fruticosum Guill. \& Perr. & $\mathrm{Ch}$ & Perennial & shrub & SA & 5 \\
\hline & Alcea acaulis (Cav.) Alef. & $\mathrm{Ch}$ & Perennial & Herb & ME & \\
\hline & Alcea striata (DC.) Alef. & $\mathrm{He}$ & Perennial & Herb & IT & \\
\hline & Malva neglecta Wallr. & Th & Annual & Herb & ME,IT & \\
\hline & Malva parviflora $\mathrm{L}$. & Th & Annual & Herb & ME,IT & \\
\hline Menispermaceae & $\begin{array}{l}\text { Cocculus pendulus (J.R.Forst. \& } \\
\text { G.Forst.) Diels }\end{array}$ & $\mathrm{Ph}$ & Perennial & Climber & SA & 1 \\
\hline \multirow[t]{2}{*}{ Moraceae } & Ficus carica $\mathrm{L}$. & $\mathrm{Ph}$ & Perennial & tree & ME,IT & \\
\hline & Ficus palmata Forssk. & $\mathrm{Ph}$ & Perennial & shrub & SU & 2 \\
\hline Moringaceae & Moringa peregrina (Forssk.) Fiori & $\mathrm{Ph}$ & Perennial & tree & SU & 1 \\
\hline Neuradaceae & Neurada procumbens L. & Th & Annual & Herb & SA & 1 \\
\hline Nitrariaceae & Peganum harmala L. & $\mathrm{He}$ & Perennial & shrub & IT,SA & 1 \\
\hline Oleaceae & Olea europaea L. & $\mathrm{Ph}$ & Perennial & tree & ME & 1 \\
\hline \multirow[t]{5}{*}{ OrobanChceae } & Cistanche salsa (C.A.Mey.) Beck & $\mathrm{Pa}$ & Perennial & Herb & IT & 5 \\
\hline & Cistanche phelypaea (L). Cout. & $\mathrm{Pa}$ & Perennial & Herb & ME,SA,IT & \\
\hline & Orobanche cernua Loefl. & $\mathrm{Pa}$ & Annual & Herb & ME,SA,IT & \\
\hline & Orobanche palaestina Reuter & pa & Annual & Herb & $\mathrm{ME}$ & \\
\hline & Lindenbergia indica (L.) Vatke. & Ch & Perennial & shrub & SA & \\
\hline Oxalidaceae & Oxalis corniculata $\mathrm{L}$. & Th & Annual & Herb & pluri-regional & 1 \\
\hline \multirow[t]{8}{*}{ Papaveraceae } & Fumaria parviflora Lam. & Th & Annual & Herb & ME,IT & 8 \\
\hline & Glaucium arabicum Fresen. & $\mathrm{He}$ & Annual & Herb & IT & \\
\hline & Glaucium flavum Crantz. & $\mathrm{He}$ & Perennial & shrub & $\mathrm{ME}$ & \\
\hline & Hypecoum pendulum L. & Th & Annual & Herb & ME,IT & \\
\hline & $\begin{array}{l}\text { Papaver decaisnei Hochst. \& Steud. ex } \\
\text { Elkan }\end{array}$ & Th & Annual & Herb & IT & \\
\hline & Papaver dodecandrum (Forssk.) Medik. & Th & Annual & Herb & ME,IT & \\
\hline & Papaver somniferum $\mathrm{L}$ & $\mathrm{He}$ & Annual & Herb & pluri-regional & \\
\hline & $\begin{array}{l}\text { Roemeria hybrida subsp. dodecandra } \\
\text { (Forssk.)Maire }\end{array}$ & Th & Annual & Herb & ME,IT & \\
\hline Phyllanthaceae & Andrachne aspera Spreng. & $\mathrm{Ch}$ & Perennial & $\begin{array}{l}\text { Herb/ } \\
\text { Subshru } \\
\text { b }\end{array}$ & SA & 1 \\
\hline \multirow[t]{6}{*}{ Plantaginaceae } & $\begin{array}{l}\text { Anarrhinum forskaohlii subsp. } \\
\text { pubescens D.A.Sutton }\end{array}$ & $\mathrm{He}$ & Perennial & shrub & $\begin{array}{l}\text { Endemic \& } \\
\text { Steno-endemic }\end{array}$ & 19 \\
\hline & Globularia arabica Jaub. \& Spach & Ch & Perennial & shrub & SA & \\
\hline & $\begin{array}{l}\text { Nanorrhinum acerbianum (Boiss.) } \\
\text { Betsche }\end{array}$ & $\mathrm{He}$ & $\begin{array}{l}\text { Annual / } \\
\text { perennial }\end{array}$ & Herb & SA & \\
\hline & $\begin{array}{l}\text { Nanorrhinum scoparium (Brouss. ex } \\
\text { Spreng.) Yousefi \& Zarre }\end{array}$ & Ch & Perennial & Herb & SA,SU & \\
\hline & Plantago afra $\mathrm{L}$. & Th & Annual & Herb & ME,SA,IT & \\
\hline & Plantago amplexicaulis Cav. & Th & Annual & Herb & ME,SA,IT & \\
\hline
\end{tabular}




\section{Floristic study of Saint Katherine protectorate, Sinai}

\begin{tabular}{|c|c|c|c|c|c|c|}
\hline Family & Accepted plant name & $\begin{array}{l}\text { life form } \\
\text { (Raunkiaer) }\end{array}$ & life spans & duration & Chorotype & $\begin{array}{c}\text { No. of } \\
\text { taxa }\end{array}$ \\
\hline & Plantago ciliata Desf. & Th & Annual & Herb & SA,IT & \\
\hline & Plantago cylindrica Forssk. & Th & $\begin{array}{l}\text { Annual / } \\
\text { perennial }\end{array}$ & Herb & SA & \\
\hline & Plantago major L. & Th & Annual & Herb & SA,IT,EU & \\
\hline & Plantago ovata Forssk. & Th & Annual & Herb & ME,SA,IT & \\
\hline & Plantago sinaica (Barnéoud) Decne. & $\mathrm{Ch}$ & Perennial & shrub & IT & \\
\hline & Plantago squarrosa Murray. & Th & Annual & Herb & $\mathrm{ME}$ & \\
\hline & Veronica anagallis-aquatica L. & He & Perennial & Herb & pluri-regional & \\
\hline & $\begin{array}{l}\text { Veronica catenata subsp. } \\
\text { pseudocatenata Chrtek \& Osb.-Kos. }\end{array}$ & $\mathrm{He}$ & Perennial & Herb & ME,EU & \\
\hline & Veronica kaiseri Täckh. & He & Perennial & Herb & $\begin{array}{l}\text { Endemic \& } \\
\text { Steno-endemic }\end{array}$ & \\
\hline & $\begin{array}{l}\text { Veronica rubrifolia subsp. } \\
\text { respectatissmia M.A. Fisch }\end{array}$ & Th & Annual & Herb & SA & \\
\hline \multirow[t]{3}{*}{ Plumbaginaceae } & Limonium lobatum (L.f.) Chz. & Th & Annual & Herb & SA & 3 \\
\hline & Limonium pruinosum (L.) Chz. & $\mathrm{Ch}$ & Perennial & Herb & SA & \\
\hline & Limonium sinuatum (L.) Mill. & $\mathrm{He}$ & Perennial & Herb & $\mathrm{ME}$ & \\
\hline \multirow[t]{27}{*}{ Poaceae } & Aegilops crassa Boiss. & Th & Annual & Herb & IT & 46 \\
\hline & $\begin{array}{l}\text { Achnatherum parviflorum (Desf.) } \\
\text { M.Nobis }\end{array}$ & $\mathrm{He}$ & Perennial & Herb & IT & \\
\hline & Aristida adscensionis L. & Th & $\begin{array}{l}\text { Annual / } \\
\text { perennial }\end{array}$ & Herb & SA,SU & \\
\hline & Arundo donax L. & $\mathrm{Ph}$ & Perennial & reeds & ME,IT & \\
\hline & Avena barbata Pott ex Link & Th & Annual & Herb & $\mathrm{ME}$ & \\
\hline & Avena fatua $\mathrm{L}$. & Th & Annual & Herb & $\mathrm{ME}$ & \\
\hline & Bromus pumilio (Trin.) P.M.Sm. & Th & Annual & Herb & ME,IT,EU & \\
\hline & Brachypodium distachyon (L.) P.Beauv. & Th & Annual & Herb & ME,IT & \\
\hline & Bromus tectorum L. & Th & Annual & Herb & ME,IT,EU & \\
\hline & Bromus catharticus Vahl & Th & $\begin{array}{l}\text { Annual / } \\
\text { perennial }\end{array}$ & Herb & ME,IT & \\
\hline & Bromus pectinatus Thunb. & Th & Annual & Herb & ME,IT & \\
\hline & Cutandia dichotoma (Forssk.) Trab. $\dagger$ & Th & Annual & Herb & SA,IT & \\
\hline & Cynodon dactylon (L.) Pers. & $\mathrm{He}$ & Perennial & Herb & pluri-regional & \\
\hline & Dactyloctenium aegyptium (L.) Willd. & Th & Annual & Herb & pluri-regional & \\
\hline & Digitaria ciliaris (Retz.)Koeler & Th & Annual & Herb & TROPICAL & \\
\hline & Digitaria sanguinalis (L.) Scop. & Th & Annual & Herb & pluri-regional & \\
\hline & Echinochloa colonum (L.) Link & Th & Annual & Herb & SA & \\
\hline & Eragrostis barrelieri Daveau & Th & Annual & Herb & ME,SA & \\
\hline & Hordeum marinum Huds. & Th & Annual & Herb & ME,IT & \\
\hline & Hyparrhenia hirta (L.) Stapf & $\mathrm{Ch}$ & Perennial & Herb & ME,SA,IT & \\
\hline & Imperata cylindrica (L.) P.Beauv. & $\mathrm{He}$ & Perennial & Herb & ME,SA,IT & \\
\hline & Lasiurus scindicus Henrard & $\mathrm{He}$ & Perennial & Herb & SA & \\
\hline & Lolium rigidum Gaudin & Th & Annual & Herb & ME,IT & \\
\hline & Lygeum spartum Loefl. ex L. & $\mathrm{He}$ & Perennial & Herb & $\mathrm{ME}$ & \\
\hline & $\begin{array}{l}\text { Melanocenchris abyssinica (R. Br. ex } \\
\text { Fresen.) Hochst. } \dagger\end{array}$ & Th & Annual & Herb & SA & \\
\hline & Melica persica Kunth. & $\mathrm{He}$ & Perennial & Herb & ME,IT & \\
\hline & $\begin{array}{l}\text { Oloptum miliaceum (L.) Röser \& } \\
\text { Hamasha }\end{array}$ & He & Perennial & Herb & ME,IT & \\
\hline
\end{tabular}




\section{Rabei et al.}

\begin{tabular}{|c|c|c|c|c|c|c|}
\hline Family & Accepted plant name & $\begin{array}{l}\text { life form } \\
\text { (Raunkiaer) }\end{array}$ & life spans & duration & Chorotype & $\begin{array}{c}\text { No. of } \\
\text { taxa }\end{array}$ \\
\hline & Panicum turgidum Forssk. & $\mathrm{Ge}$ & Perennial & Herb & ME,SA,IT & \\
\hline & Phlaris minor Retz. & Th & Annual & Herb & ME,IT & \\
\hline & $\begin{array}{l}\text { Phragmites australis (Cav.) Trin. ex } \\
\text { Steud. }\end{array}$ & $\mathrm{He}$ & Perennial & reeds & pluri-regional & \\
\hline & $\begin{array}{l}\text { Piptatherum holciforme (M.Bieb.) } \\
\text { Roem. \& Schult. }\end{array}$ & $\mathrm{He}$ & Perennial & Herb & ME,IT & \\
\hline & Poa sinaica Steud. & $\mathrm{He}$ & Perennial & Herb & IT & \\
\hline & Polypogon monspeliensis (L.) Desf. & Th & Annual & Herb & ME,SA,IT & \\
\hline & Schismus barbatus (L.) Thell. & Th & Annual & Herb & SA,IT,EU & \\
\hline & Setaria verticillata (L.) P. Beauv. & Th & Annual & Herb & pluri-regional & \\
\hline & Setaria viridis (L.) P. Beauv. & Th & Annual & Herb & ME,IT & \\
\hline & Sorghum virgatum (Hack.) Stapf & $\mathrm{Ch}$ & Annual & Herb & IT,SA,ME,SU & \\
\hline & Stipa arabica Trin. \& Rupr. & He & Perennial & Herb & IT & \\
\hline & $\begin{array}{l}\text { Stipellula capensis (Thunb.) Röser \& } \\
\text { Hamasha }\end{array}$ & Th & Annual & Herb & IT,SA & \\
\hline & Stipagrostis ciliata (Desf.) De Winter & He & Perennial & Herb & SA & \\
\hline & $\begin{array}{l}\text { Stipagrostis plumosa (L.) Munro ex } \\
\text { T.Anderson }\end{array}$ & $\mathrm{He}$ & Perennial & Herb & IT,SA & \\
\hline & Stipagrostis raddiana (Savi) De Winter & He & Perennial & Herb & SA & \\
\hline & $\begin{array}{l}\text { Taeniatherum caput-medusae (L.) } \\
\text { Nevski. }\end{array}$ & Th & Annual & Herb & IT & \\
\hline & Tetrapogon villosus Desf. & Ge & Perennial & Herb & SA & \\
\hline & Tricholaena teneriffae (L. f.) Link & $\mathrm{Ge}$ & Perennial & Herb & ME,SA,IT & \\
\hline Polygalaceae & Polygala Sinaica Botsch. & $\mathrm{Ch}$ & Perennial & shrub & $\begin{array}{l}\text { Endemic \& } \\
\text { Steno-endemic }\end{array}$ & 1 \\
\hline \multirow[t]{3}{*}{ Polygonaceae } & AtraPhxis spinosa L. & $\mathrm{Ch}$ & Perennial & shrub & IT & 3 \\
\hline & Calligonum comosum L'Hér. & $\mathrm{Ph}$ & Perennial & shrub & SA,IT & \\
\hline & Rumex vesicarius L. & Th & Annual & Herb & ME,SA,IT & \\
\hline Portulacaceae & Portulaca oleracea L. & Th & Annual & Herb & pluri-regional & 1 \\
\hline \multirow[t]{2}{*}{ Primulaceae } & $\begin{array}{l}\text { Lysimachia arvensis (L.) U.Manns \& } \\
\text { Anderb. }\end{array}$ & Th & Annual & Herb & ME,IT & \\
\hline & $\begin{array}{l}\text { Primula involucrata (Raf.) Link \& Otto } \\
\text { ex Sweet }\end{array}$ & He & Perennial & Herb & $\begin{array}{l}\text { Endemic \& } \\
\text { Steno-endemic }\end{array}$ & 2 \\
\hline \multirow[t]{2}{*}{ Pteridaceae } & Adiantum capillus-veneris L. & He & Perennial & Herb & ME,IT & \\
\hline & $\begin{array}{l}\text { Heionitis pteridioides (ReiChrd) } \\
\text { Christenh. } \dagger\end{array}$ & $\mathrm{He}$ & Perennial & Herb & ME & 2 \\
\hline \multirow[t]{6}{*}{ Resedaceae } & $\begin{array}{l}\text { Caylusea hexagyna (Forssk.) M. L. } \\
\text { Green. }\end{array}$ & Th & $\begin{array}{l}\text { Annual / } \\
\text { perennial }\end{array}$ & Herb & SU & 6 \\
\hline & Ochradenus baccatus Delile & $\mathrm{Ph}$ & Perennial & shrub & SU & \\
\hline & $\begin{array}{l}\text { Oligomeris linifolia (Vahl ex Hornem.) } \\
\text { J.F.Macbr. }\end{array}$ & Th & Annual & Herb & SU & \\
\hline & Reseda muricata C. Presl & $\mathrm{Ch}$ & Perennial & Herb & SA & \\
\hline & Reseda pruinosa Delile & He & Annual & Herb & IT,SA & \\
\hline & Reseda stenostachya Boiss. & $\mathrm{Ch}$ & Annual & Herb & SA & \\
\hline \multirow[t]{2}{*}{ Rhamnaceae } & Rhamnus disperma Ehrenb. ex Boiss. & $\mathrm{Ph}$ & Perennial & shrub & SA & 2 \\
\hline & Ziziphus spina-christi (L.) Desf. & $\mathrm{Ph}$ & Perennial & tree & SU & \\
\hline \multirow[t]{3}{*}{ Rosaceae } & Cotoneaster orbicularis Schltdl. & $\mathrm{Ph}$ & Perennial & shrub & SA & 4 \\
\hline & Crataegus $x$ sinaica Boiss. & $\mathrm{Ph}$ & Perennial & tree & ME,IT & \\
\hline & Rosa arabica (Crép. ex Boiss.) Déségl. & $\mathrm{Ph}$ & Perennial & shrub & $\begin{array}{l}\text { Endemic \& } \\
\text { Steno-endemic }\end{array}$ & \\
\hline
\end{tabular}




\section{Floristic study of Saint Katherine protectorate, Sinai}

\begin{tabular}{|c|c|c|c|c|c|c|}
\hline Family & Accepted plant name & $\begin{array}{l}\text { life form } \\
\text { (Raunkiaer) }\end{array}$ & life spans & duration & Chorotype & $\begin{array}{c}\text { No. of } \\
\text { taxa }\end{array}$ \\
\hline & Rubus creticus Tourn. ex L. & $\mathrm{Ph}$ & Perennial & shrub & ME,IT & \\
\hline \multirow[t]{8}{*}{ Rubiaceae } & Callipeltis cucullaris (L.) DC. $\dagger$ & Th & Annual & Herb & ME,SA,IT & 8 \\
\hline & Crucianella ciliata Lam. & Th & Annual & Herb & IT & \\
\hline & Galium aparine L. & Th & Annual & Herb & ME,IT & \\
\hline & Galium ceratopodum Boiss. & Th & Annual & Herb & ME & \\
\hline & Galium setaceum Lam. & Th & Annual & Herb & IT & \\
\hline & $\begin{array}{l}\text { Galium sinaicum (Delile ex Decne.) } \\
\text { Boiss. }\end{array}$ & $\mathrm{Ch}$ & Perennial & Herb & ME,SA,IT & \\
\hline & Kohautia caespitosa subsp. caespitosa & Th & $\begin{array}{l}\text { Annual / } \\
\text { perennial }\end{array}$ & Herb & SA,SU & \\
\hline & $\begin{array}{l}\text { Plocama calycoptera (Decne.) } \\
\text { M.Backlund \& Thulin }\end{array}$ & $\mathrm{Ch}$ & Perennial & shrub & SA & \\
\hline Rutaceae & $\begin{array}{l}\text { Haplophyllum tuberculatum (Forssk.) } \\
\text { A.Juss. }\end{array}$ & $\mathrm{Ch}$ & Perennial & Herb & SA & 1 \\
\hline Salicaceae & Salix mucronata Thunb. & $\mathrm{Ph}$ & Perennial & tree & ME,IT & 1 \\
\hline \multirow[t]{6}{*}{ Scrophulariaceae } & Scrophularia deserti Delile. & $\mathrm{He}$ & Perennial & Herb & SA & 6 \\
\hline & Scrophularia libanotica Boiss. & $\mathrm{He}$ & Perennial & Herb & $\mathrm{ME}$ & \\
\hline & Scrophularia xanthogolssa Boiss. & $\mathrm{Ch}$ & Perennial & Herb & ME,IT & \\
\hline & Verbascum decaisneanum Kuntze & $\mathrm{Ch}$ & Perennial & Herb & SA & \\
\hline & Verbascum sinaiticum Benth. & $\mathrm{He}$ & Biennial & Herb & ME,SA,IT & \\
\hline & Verbascum sinuatum L. & $\mathrm{He}$ & Biennial & Herb & ME,IT & \\
\hline \multirow[t]{11}{*}{ Solanaceae } & Datura ferox $\mathrm{L}$. & Th & Annual & Herb & SubT & 12 \\
\hline & Datura innoxia Mill. & Th & Annual & Herb & pluri-regional & \\
\hline & $\begin{array}{l}\text { Hyoscyamus boveanus (Dunal) Asch. \& } \\
\text { Schweinf. }\end{array}$ & $\mathrm{He}$ & Perennial & Herb & $\begin{array}{l}\text { Endemic \& } \\
\text { Steno-endemic }\end{array}$ & \\
\hline & $\begin{array}{l}\text { Hyoscyamus desertorum (Asch. Ex } \\
\text { Boiss.) Täckh. }\end{array}$ & $\mathrm{He}$ & Annual & Herb & $\mathrm{ME}$ & \\
\hline & Hyoscyamus muticus L. $†$ & $\mathrm{He}$ & Perennial & shrub & SA,IT & \\
\hline & Hyoscyamus pusillus L. & Th & Annual & Herb & IT & \\
\hline & Lycium shawii Roem. \& Schult. & $\mathrm{Ph}$ & Perennial & shrub & SA,SU & \\
\hline & Nicotiana glauca Graham & $\mathrm{Ph}$ & Perennial & shrub & pluri-regional & \\
\hline & Solanum nigrum L. & $\mathrm{He}$ & Annual & Herb & ME,IT & \\
\hline & Solanum villosum Mill. & $\mathrm{Ch}$ & Perennial & Herb & SA & \\
\hline & Withania somnifera (L.) Dunal & $\mathrm{Ch}$ & Perennial & shrub & ME,IT & \\
\hline \multirow[t]{3}{*}{ Tamaricaceae } & Reaumuria alternifolia (Labill.)Britten & $\mathrm{Ch}$ & Perennial & shrub & IT,SA & 3 \\
\hline & Tamarix aphylla (L.) H. Karst. & $\mathrm{Ph}$ & Perennial & tree & SU & \\
\hline & Tamarix nilotica (Ehrenb.) Bunge & $\mathrm{Ph}$ & Perennial & tree & SA & \\
\hline TyPhceae & TyPh domingensis Pers. & На & Perennial & Herb & ME,SA,IT & 1 \\
\hline \multirow[t]{2}{*}{ Urticaceae } & Forsskaolea tenacissima L. & $\mathrm{Ch}$ & Perennial & shrub & SA,SU & 2 \\
\hline & Parietaria alsinifolia Delile & Th & Annual & Herb & SA,IT & \\
\hline Verbenaceae & Phyla nodiflora (L.) Greene. & $\mathrm{He}$ & Perennial & Herb & ME,TOPICAL & 1 \\
\hline \multirow[t]{4}{*}{ Zygophyllaceae } & Tribulus pentandrus Forssk. & Th & Annual & Herb & SA,SU & 7 \\
\hline & Tribulus terrestris L. & Th & Annual & Herb & ME,IT & \\
\hline & $\begin{array}{l}\text { Zygophyllum arabicum (L.) Christenh. \& } \\
\text { Byng }\end{array}$ & $\mathrm{Ch}$ & Perennial & $\begin{array}{l}\text { sub } \\
\text { Shrub }\end{array}$ & SA & \\
\hline & $\begin{array}{l}\text { Zygophyllum bruguieri (DC.) Christenh. } \\
\text { \& Byng }\end{array}$ & $\mathrm{He}$ & Perennial & $\begin{array}{l}\text { sub } \\
\text { Shrub }\end{array}$ & SA,IT & \\
\hline
\end{tabular}




\section{Rabei et al.}

\begin{tabular}{llllll}
\hline Family & Accepted plant name & $\begin{array}{l}\text { life form } \\
\text { (Raunkiaer) }\end{array}$ & life spans & duration & $\begin{array}{c}\text { Chorotype } \\
\text { No. of } \\
\text { taxa }\end{array}$ \\
\hline & Zygophyllum coccineum L. & Ch & Perennial & shrub & SA \\
& $\begin{array}{l}\text { Zygophyllum molle (Delile) Christenh. \& } \\
\text { Byng }\end{array}$ & Ch & Perennial & sub & SA \\
Zygophyllum simplex L. & Th & $\begin{array}{l}\text { Annual / } \\
\text { bienniaal }\end{array}$ & Herb & SA,SU \\
\hline
\end{tabular}

